CERN-TH.7487/94

hep-ph/9412265

\title{
Scale Setting in QCD and the Momentum Flow in Feynman Diagrams
}

\author{
Matthias Neubert \\ Theory Division, CERN, CH-1211 Geneva 23, Switzerland
}

\begin{abstract}
We present a formalism to evaluate QCD diagrams with a single virtual gluon using a running coupling constant at the vertices. This method, which corresponds to an all-order resummation of certain terms in a perturbative series, provides a description of the momentum flow through the gluon propagator. It can be viewed as a generalization of the scale-setting prescription of Brodsky, Lepage and Mackenzie to all orders in perturbation theory. In particular, the approach can be used to investigate why in some cases the "typical" momenta in a loop diagram are different from the "natural" scale of the process. It offers an intuitive understanding of the appearance of infrared renormalons in perturbation theory and their connection to the rate of convergence of a perturbative series. Moreover, it allows one to separate short- and long-distance contributions by introducing a hard factorization scale. Several applications to one- and two-scale problems are discussed in detail.
\end{abstract}

CERN-TH.7487/94

December 1994 


\section{Introduction}

Perturbative expansions in field theory are asymptotic, making it necessary from a conceptual point of view (if not from a practical one) to truncate any perturbative series at some finite order in the coupling constant. This truncation introduces renormalization-scale and scheme dependences. In QCD, this poses a problem because of the strong scale dependence of the running coupling constant $\alpha_{s}\left(\mu^{2}\right)$ in the low-momentum region. In many cases this puts limitations on the precision with which experimental data can be described in terms of the fundamental theory of strong interactions.

Several prescriptions on how to fix the renormalization scale and scheme in a truncated perturbative series have been proposed [1]-10]. They all rely, in some way or another, on a guess about the size of uncalculated higher-order contributions. This guess can be based on criteria such as the apparent rate of convergence of a series, the size of the coefficient of the last term in the truncated series, or the sensitivity to changes of the renormalization scale and scheme. It can also rely on physical criteria such as the role of various mass scales in a given problem. An example for such a physical scheme is provided by the scale-setting prescription of Brodsky, Lepage and Mackenzie (BLM), which amounts to absorb certain vacuum polarization effects appearing at two-loop order into the one-loop running coupling constant [4]. Extensions of this scheme beyond the one-loop order have been considered in Refs. [10]-12].

A large higher-order coefficient in a perturbative series can arise from an anomalously large contribution of a particular set of higher-order diagrams. For instance, one can imagine a series with a large two-loop coefficient, but small oneloop and higher-order coefficients. Such "genuine" higher-order effects are hard to anticipate without a detailed calculation. On the other hand, it is possible that large higher-order corrections result from an inappropriate choice of the renormalization scale or scheme. In particular, a change of the scale in the running coupling constant at one-loop order leads to a change proportional to $\beta_{0} \alpha_{s}^{2}$ at two-loop order, where $\beta_{0}=11-\frac{2}{3} n_{f}$ is the first coefficient of the $\beta$-function, and $n_{f}$ denotes the number of light quark flavours. Since $\beta_{0}$ is large in QCD, such effects can be numerically significant. An appropriate choice of scale should try to minimize this type of higher-order terms. Surprisingly, however, one finds that even in one-scale problems using the "natural" scale in the running coupling constant at one-loop order often leaves large corrections of order $\beta_{0} \alpha_{s}^{2}$. Let us consider some examples related to heavy quark systems: (i) the relation between the pole mass $m_{b}$ of the bottom quark and the mass $\bar{m}_{b}\left(m_{b}\right)$ renormalized in the modified minimal subtraction $(\overline{\mathrm{MS}})$ scheme; (ii) the ratio of the decay constants of pseudoscalar and vector mesons, $B$ and $B^{*}$, in the so-called static limit; (iii) the parton model prediction for the semi-leptonic decay rate $\Gamma\left(b \rightarrow u e \bar{\nu}_{e}\right)$. At 
two-loop order, the corresponding perturbative series are [13]-15]

$$
\begin{aligned}
\frac{m_{b}}{\bar{m}_{b}\left(m_{b}\right)} & =1+\frac{4}{3} \frac{\alpha_{s}\left(m_{b}^{2}\right)}{\pi}+\left(1.56 \beta_{0}-1.05\right)\left(\frac{\alpha_{s}\left(m_{b}^{2}\right)}{\pi}\right)^{2}+\ldots, \\
\frac{f_{B}^{\text {stat }}}{f_{B^{*}}^{\text {stat }}} & =1+\frac{2}{3} \frac{\alpha_{s}\left(m_{b}^{2}\right)}{\pi}+\left(0.53 \beta_{0}+2.41\right)\left(\frac{\alpha_{s}\left(m_{b}^{2}\right)}{\pi}\right)^{2}+\ldots, \\
\frac{\Gamma\left(b \rightarrow u e \bar{\nu}_{e}\right)}{\Gamma_{\text {tree level }}} & =1-2.41 \frac{\alpha_{s}\left(m_{b}^{2}\right)}{\pi}-\left(3.22 \beta_{0}+k\right)\left(\frac{\alpha_{s}\left(m_{b}^{2}\right)}{\pi}\right)^{2}+\ldots,
\end{aligned}
$$

where the constant $k$ is yet unknown. In all cases the running coupling constant is renormalized in the $\overline{\mathrm{MS}}$ scheme. For $n_{f}=4$, the parts of the two-loop coefficients proportional to $\beta_{0}$ are $13.02,4.40$, and 26.08, respectively. At least in the first two examples these terms provide the dominant part of the two-loop corrections. Moreover, in all three cases the two-loop corrections are quite large, casting doubt on the convergence of the perturbative series. An important example related to light quarks is the so-called $D$-function, which is proportional to the derivative of the correlator of two vector currents containing massless quark fields. At two-loop order, one finds [16]-18

$$
D\left(Q^{2}\right)=1+\frac{\alpha_{s}\left(Q^{2}\right)}{\pi}+\left(0.17 \beta_{0}+0.08\right)\left(\frac{\alpha_{s}\left(Q^{2}\right)}{\pi}\right)^{2}+\ldots,
$$

where $Q^{2}$ denotes the euclidean momentum transfer. Again, the term proportional to $\beta_{0}$ dominates the two-loop coefficient. However, the absolute size of the two-loop correction is smaller than in the cases considered above.

The appearance of large corrections proportional to $\beta_{0} \alpha_{s}^{2}$ can be interpreted as an inappropriate choice of scale in the one-loop running coupling constant. BLM have argued that one should absorb these terms, which arise from selfenergy corrections to the gluon propagator, into the one-loop running coupling constant. This prescription defines the so-called BLM scale $\mu_{\mathrm{BLM}}$. For the above examples, one finds (in the $\overline{\mathrm{MS}}$ scheme): $\mu_{\mathrm{BLM}}=0.10 m_{b}, 0.21 m_{b}, 0.07 m_{b}$, and $0.71 \sqrt{Q^{2}}$. Clearly, if the BLM scale is to be interpreted as a "typical" scale of virtual momenta in the corresponding Feynman diagrams, the question arises why it is often much lower than the "natural" mass scale in the problem at hand.

To analyse this issue, we propose a generalization of the BLM prescription. Consider the perturbative calculation of a physical (i.e. renormalization-scheme invariant and infrared finite) quantity $S\left(M^{2}\right)$, which depends upon a single large mass scale $M$. We restrict our discussion to Green functions without external gluons. A generalization of the method to physical cross sections and inclusive decay rates, which receive both virtual and real gluon corrections, will be presented elsewhere [19]. The aim of the BLM prescription is to "guess" the average virtuality of the gluon in a loop diagram and to use it as the scale in the running coupling constant. Clearly, a better way to proceed would be to perform 
the calculation with a running coupling constant $\alpha_{s}\left(-k^{2}\right)$ at the vertices, where $k$ is the momentum flowing through the virtual gluon line. The result of such an improved calculation, which we denote by $S_{\text {res }}\left(M^{2}\right)$ since it corresponds to a partial resummation of the perturbative series (see below), may be written as

$$
S_{\text {res }}\left(M^{2}\right)=\int \mathrm{d}^{4} k \alpha_{s}\left(-k^{2}\right) f(k, M, \ldots) \equiv \int_{0}^{\infty} \mathrm{d} t w(t) \frac{\alpha_{s}\left(t M^{2}\right)}{4 \pi},
$$

where $f(k, M, \ldots)$ is the integrand of the Feynman diagrams. The function $w(t)$ describes the distribution of virtualities in the loop calculation. The integral over this distribution function corresponds to an average of the running coupling constant over the loop momenta in one-loop diagrams and, in a way, provides the optimal improvement that can be achieved without a complete higher-order calculation.

The fact that the integration in (3) extends to $t \rightarrow 0$ indicates the appearance of non-perturbative effects. It makes explicit that any perturbative calculation receives long-distance contributions from the integration over low momenta in Feynman diagrams. We shall discuss the significance of these contributions at length below. Here we just note that our approach goes beyond perturbation theory since it is equivalent to the resummation of an infinite number of terms in the perturbative series for the quantity $S\left(M^{2}\right)$. Let us write this series in the general form

$$
S_{\text {pert }}\left(M^{2}\right)=\sum_{n=1}^{\infty}\left(\frac{\alpha_{s}\left(M^{2}\right)}{4 \pi}\right)^{n} S_{n} .
$$

The expansion coefficients $S_{n}$ can be written as a power series in the number of light quark flavours or, equivalently, as a series in powers of $\beta_{0}$. We restrict ourselves to cases where the calculation of the one-loop coefficient $S_{1}$ does not involve the non-abelian gluon self couplings, and where there are no external gluons. Then the first dependence on $n_{f}$ comes at two-loop order, and in general the coefficients can be written in the form

$$
S_{n}=c_{n} \beta_{0}^{n-1}+d_{n} \beta_{0}^{n-2}+\ldots+k_{n}=c_{n}\left(-\frac{2}{3} n_{f}\right)^{n-1}+O\left(n_{f}^{n-2}\right) .
$$

The coefficient $c_{n}$ in front of the highest power of $\beta_{0}$ is proportional to the coefficient in front of the highest power of $n_{f}$. This implies that $c_{n}$ can be calculated from "quasi one-loop" diagrams, in which a gluon line is dressed by $(n-1)$ lightquark loops. It is the partial series built up by the terms proportional to $c_{n}$ which is resummed in our approach. To see this, we use the one-loop renormalizationgroup equation for the running coupling constant to relate $\alpha_{s}\left(t M^{2}\right)$ in (3) to $\alpha_{s}\left(M^{2}\right)$ :

$$
\alpha_{s}\left(t M^{2}\right)=\alpha_{s}\left(M^{2}\right) \sum_{n=1}^{\infty}\left(\frac{\beta_{0} \alpha_{s}\left(M^{2}\right)}{4 \pi}\right)^{n-1}(-\ln t)^{n-1}
$$


Under the above assumptions it is easy to see that the running of the coupling constant is the only source of terms of order $\beta_{0}^{n-1} \alpha_{s}^{n}$ in the series (雨). We thus conclude that

$$
S_{\mathrm{res}}\left(M^{2}\right)=\int_{0}^{\infty} \mathrm{d} t w(t) \frac{\alpha_{s}\left(t M^{2}\right)}{4 \pi}=\sum_{n=1}^{\infty}\left(\frac{\alpha_{s}\left(M^{2}\right)}{4 \pi}\right)^{n} c_{n} \beta_{0}^{n-1} .
$$

The BLM prescription is to absorb the two-loop term $c_{2} \beta_{0} \alpha_{s}^{2}$ into a redefinition of the scale used in the coupling constant at one-loop order. It is equivalent to setting $w_{\mathrm{BLM}}(t)=c_{1} \delta\left(t-e^{-c_{2} / c_{1}}\right)$, thus choosing an average virtuality $\mu_{\mathrm{BLM}}^{2}=$ $\exp \left(-c_{2} / c_{1}\right) M^{2}$ for the gluon. Already at this point it is clear that this can only be a good approximation if the distribution function $w(t)$ is narrow. If it is wide, it is better to perform the integral in (3), which resums all terms of the form $c_{n} \beta_{0}^{n-1} \alpha_{s}^{n}$.

Clearly, the resummation in (3) does not replace a full higher-order calculation. For instance, "genuine" two-loop corrections not related to the running of the coupling constant are not taken into account. Nevertheless, our approach can be considered as an optimal improvement of one-loop calculations, which takes into account the full information contained in one-loop diagrams combined with the running of the coupling constant. As such, we believe that the construction of the distribution function $w(t)$ is an interesting new concept, which provides information that goes beyond what is contained in a low-order perturbative calculation.

In Sect. 2 we generalize the above discussion in such a way that the scale and scheme independence of the procedure become apparent. In Sect. 3 we discuss the asymptotic behaviour of the distribution function and its relation to the ultraviolet and infrared properties of the perturbative series. In particular, we trace the appearance of infrared renormalons and discuss how to separate shortand long-distance contributions by introducing a hard factorization scale. In Sects. 1 and 5 we calculate the distribution function for several one- and twoscale problems, among them most of the quantities considered in (1) and (2). We present a detailed numerical analysis, in which we compare the results for the resummed series to low-order calculations and investigate the relative size of short- and long-distance contributions. Section 6 contains a summary and conclusions.

\section{Construction of the distribution function}

We will now repeat the above argument in a slightly more general form, which allows us to keep track of scale and scheme dependence. Consider some dimensionless, infrared-safe and renormalization-scheme invariant quantity $S\left(M^{2}, z\right)$, which depends upon some large mass scale $M$ and, in the most general case, on 
a set of dimensionless parameters $z$. For instance, in a two-scale problem we may choose $M^{2}=m_{1} m_{2}$ and $z=m_{2} / m_{1}$. Let us investigate the perturbative series of $S\left(M^{2}, z\right)$ in powers of the coupling constant $\alpha_{s}^{\mathrm{R}}\left(\mu^{2}\right)$ renormalized at some scale $\mu$ and in some renormalization scheme $\mathrm{R}$ :

$$
S_{\text {pert }}\left(M^{2}, z\right)=\sum_{n=1}^{\infty}\left(\frac{\alpha_{s}^{\mathrm{R}}\left(\mu^{2}\right)}{4 \pi}\right)^{n} S_{n}^{\mathrm{R}}(\mu, M, z) .
$$

The scale and scheme dependence in (8) cancels between the coefficient functions and the coupling constant. As in (5), the coefficients can be expanded in powers of $\beta_{0}$,

$$
S_{n}^{\mathrm{R}}(\mu, M, z)=c_{n}^{\mathrm{R}}(\mu, M, z) \beta_{0}^{n-1}+\ldots,
$$

and we define a function $w_{\mathrm{R}}(t, \mu, M, z)$ such that

$$
S_{\mathrm{res}}\left(M^{2}, z\right)=\int_{0}^{\infty} \mathrm{d} t w_{\mathrm{R}}(t, \mu, M, z) \frac{\alpha_{s}^{\mathrm{R}}\left(t \mu^{2}\right)}{4 \pi}=\sum_{n=1}^{\infty}\left(\frac{\alpha_{s}^{\mathrm{R}}\left(\mu^{2}\right)}{4 \pi}\right)^{n} c_{n}^{\mathrm{R}}(\mu, M, z) \beta_{0}^{n-1} .
$$

Using (6) and the fact that, by construction, the distribution function must be independent of $n_{f}$, one can derive a relation between the moments of the distribution function and the coefficients $c_{n}^{\mathrm{R}}$. It reads

$$
\int_{0}^{\infty} \mathrm{d} t w_{\mathrm{R}}(t, \mu, M, z)(-\ln t)^{n-1}=c_{n}^{\mathrm{R}}(\mu, M, z) ; \quad n \geq 1 .
$$

By inverting this relation one can in principle obtain the distribution function from the knowledge of the set of coefficients $\left\{c_{n}^{\mathrm{R}}\right\}$. This observation is crucial, as it implies that in order to construct the distribution function it is sufficient to consider the perturbative series (8) in the fictitious limit $\beta_{0} \rightarrow \infty$ (or $\left.n_{f} \rightarrow-\infty\right)$.

To proceed, it is convenient to construct a generating function for the coefficients $c_{n}^{\mathrm{R}}$, which we define as

$$
\widetilde{S}_{\mathrm{R}}(u, \mu, M, z)=\sum_{n=1}^{\infty} \frac{u^{n-1}}{\Gamma(n)} c_{n}^{\mathrm{R}}(\mu, M, z),
$$

so that

$$
c_{n}^{\mathrm{R}}(\mu, M, z)=\left.\left(\frac{\mathrm{d}}{\mathrm{d} u}\right)^{n-1} \widetilde{S}_{\mathrm{R}}(u, \mu, M, z)\right|_{u=0} .
$$

This generating function is the Borel transform of the series (8) with respect to the inverse coupling constant [20], in the limit $\beta_{0} \rightarrow \infty$. Eq. (12) can be formally inverted to give

$$
S_{\mathrm{res}}\left(M^{2}, z\right)=\frac{1}{\beta_{0}} \int_{0}^{\infty} \mathrm{d} u \widetilde{S}_{\mathrm{R}}(u, \mu, M, z) \exp \left(-\frac{4 \pi u}{\beta_{0} \alpha_{s}^{\mathrm{R}}\left(\mu^{2}\right)}\right) .
$$


In cases where the integral exists, this equation defines the Borel sum of the partial series $S_{\text {res }}\left(M^{2}, z\right)$. In general, however, the Borel transform has singularities on the real $u$-axis, corresponding a factorial growth of the expansion coefficients $c_{n}^{\mathrm{R}}$. Much of the non-perturbative structure of QCD can be inferred from a study of the Borel transform [20]-27]. Its singularities on the negative axis arise from the large-momentum region in Feynman diagrams and are called ultraviolet renormalons. They are Borel summable and pose no problem to performing the Laplace integral in (14). Singularities on the positive axis arise from the lowmomentum region in Feynman diagrams and are called infrared renormalons. Their presence leads to an ambiguity in the evaluation of the Laplace integral. In the following section we will discuss how this ambiguity is reflected in the integral over the distribution function in (3).

The Borel transform defined in (12) can be calculated by evaluating one-loop diagrams in which the gluon propagator in Landau gauge is replaced by 27]

$$
D_{a b}^{\mu \nu}(k)=i \delta_{a b}\left(\frac{e^{C}}{\mu^{2}}\right)^{-u} \frac{k^{\mu} k^{\nu}-g^{\mu \nu} k^{2}}{\left(-k^{2}\right)^{2+u}},
$$

where $C$ is a scheme-dependent constant related to the finite part of a renormalized fermion-loop insertion on the gluon propagator. Since we have assumed that the expansion coefficients $c_{n}^{\mathrm{R}}$ are dimensionless, it follows that the Borel transform can be factorized as

$$
\widetilde{S}_{\mathrm{R}}(u, \mu, M, z)=\left(\frac{e^{C} M^{2}}{\mu^{2}}\right)^{-u} \widehat{S}(u, z),
$$

where the new function $\widehat{S}(u, z)$ is scale- and scheme-independent. From (11) and (12), we find that the Borel transform can be expressed in terms of the distribution function by the integral relation

$$
\widetilde{S}_{\mathrm{R}}(u, \mu, M, z)=\int_{0}^{\infty} \mathrm{d} t w_{\mathrm{R}}(t, \mu, M, z) t^{-u},
$$

which can be inverted to give

$$
w_{\mathrm{R}}(t, \mu, M, z)=\frac{1}{2 \pi i t} \int_{u_{0}-i \infty}^{u_{0}+i \infty} \mathrm{d} u \widehat{S}(u, z)\left(\frac{t \mu^{2}}{e^{C} M^{2}}\right)^{u} .
$$

The choice of $u_{0}$ is arbitrary provided the integral exists. It follows that the distribution density $w_{R} \mathrm{~d} t$ depends on $t, \mu$ and $M$ only in the combination

$$
\tau=\frac{t \mu^{2}}{e^{C} M^{2}}
$$


If we introduce $\tau$ as a new variable and define a new scale- and scheme-independent function $\widehat{w}(\tau, z)$ so that

$$
\begin{aligned}
\widehat{w}(\tau, z) & =\frac{1}{2 \pi i} \int_{u_{0}-i \infty}^{u_{0}+i \infty} \mathrm{d} u \widehat{S}(u, z) \tau^{u-1}, \\
\widehat{S}(u, z) & =\int_{0}^{\infty} \mathrm{d} \tau \widehat{w}(\tau, z) \tau^{-u}, \\
c_{n}^{\mathrm{R}}(1, z) & =\int_{0}^{\infty} \mathrm{d} \tau \widehat{w}(\tau, z)(-C-\ln \tau)^{n-1},
\end{aligned}
$$

eq. (10) can be written in a form that makes explicit the renormalization-scheme invariance of the perturbative series:

$$
S_{\mathrm{res}}\left(M^{2}, z\right)=\int_{0}^{\infty} \mathrm{d} \tau \widehat{w}(\tau, z) \frac{\alpha_{s}\left(\tau e^{C} M^{2}\right)}{4 \pi}=\sum_{n=1}^{\infty}\left(\frac{\alpha_{s}^{\mathrm{R}}\left(\mu^{2}\right)}{4 \pi}\right)^{n} c_{n}^{\mathrm{R}}(\mu, M, z) \beta_{0}^{n-1} .
$$

Note that the scheme-dependence of the constant $C$ is such that the value of the coupling constant $\alpha_{s}\left(e^{C} \mu^{2}\right)$ is scheme-independent. This implies that the product $e^{-C / 2} \Lambda_{\mathrm{QCD}}$ is scheme-independent, where $\Lambda_{\mathrm{QCD}}$ is the scale parameter in the oneloop expression for the running coupling constant. We note that $C=-5 / 3$ in the $\overline{\mathrm{MS}}$ scheme, $C=-5 / 3+\gamma-\ln 4 \pi$ in the MS scheme, and $C=0$ in the so-called $\mathrm{V}$ scheme 4 .

As pointed out in the introduction, the integral over the distribution function in (21) is an improved one-loop approximation to the quantity $S\left(M^{2}, z\right)$. It is instructive to compare this approximation to the BLM scale-setting prescription. We find

$$
\begin{aligned}
S_{\mathrm{res}}(M, z) & =\int_{0}^{\infty} \mathrm{d} \tau \widehat{w}(\tau, z) \frac{\alpha_{s}\left(\tau e^{C} M^{2}\right)}{4 \pi}=N\left\langle\frac{\alpha_{s}\left(\tau e^{C} M^{2}\right)}{\pi}\right\rangle \\
& =N \frac{\alpha_{s}\left(\mu_{\mathrm{BLM}}^{2}\right)}{\pi}\left\{1+\Delta\left(\frac{\beta_{0} \alpha_{s}\left(\mu_{\mathrm{BLM}}^{2}\right)}{4 \pi}\right)^{2}+\ldots\right\},
\end{aligned}
$$

where

$$
\begin{aligned}
N & =\frac{1}{4} \int_{0}^{\infty} \mathrm{d} \tau \widehat{w}(\tau, z), \\
\mu_{\mathrm{BLM}}^{2} & =\exp (\langle\ln \tau\rangle+C) M^{2}, \\
\Delta & =\sigma_{\tau}^{2}=\left\langle\ln ^{2} \tau\right\rangle-\langle\ln \tau\rangle^{2} .
\end{aligned}
$$


We use the symbol

$$
\langle f(\tau)\rangle=\frac{\int_{0}^{\infty} \mathrm{d} \tau \widehat{w}(\tau, z) f(\tau)}{\int_{0}^{\infty} \mathrm{d} \tau \widehat{w}(\tau, z)}
$$

for the average of a function $f(\tau)$ over the distribution $\widehat{w}(\tau, z)$. Both the value of the coupling constant $\alpha_{s}\left(\mu_{\mathrm{BLM}}^{2}\right)$ and the parameter $\Delta$ are renormalizationscheme invariant. We observe that the first correction to the BLM scheme is related to the width of the distribution function. Note, however, that in some cases the distribution function is not of a definite sign and has no probabilistic interpretation. Thus, it may happen that $\sigma_{\tau}^{2}$ is negative or that the normalization integral $N$ vanishes, in which case $\langle f(\tau)\rangle$ would be ill-defined. If the distribution function has a definite sign, on the other hand, the quantity

$$
\delta_{\mathrm{BLM}}=\Delta\left(\frac{\beta_{0} \alpha_{s}\left(\mu_{\mathrm{BLM}}^{2}\right)}{4 \pi}\right)^{2}=\frac{\left\langle\ln ^{2} \tau\right\rangle-\langle\ln \tau\rangle^{2}}{\left[\ln \left(M^{2} / \Lambda_{\mathrm{V}}^{2}\right)+\langle\ln \tau\rangle\right]^{2}},
$$

provides a measure of the rate of convergence of the perturbative series. Here

$$
\Lambda_{\mathrm{V}}=e^{-C / 2} \Lambda_{\mathrm{QCD}}=e^{5 / 6} \Lambda_{\overline{\mathrm{MS}}}
$$

is a scheme-independent parameter, which coincides with the QCD scale parameter in the $\mathrm{V}$ scheme.

At this point it is worthwhile to point out the advantages of our resummation

over the BLM prescription. In our approach all terms of order $\beta_{0}^{n-1} \alpha_{s}^{n}$ in the perturbative series are resummed exactly, whereas the BLM scheme only resums the two-loop term of order $\beta_{0} \alpha_{s}^{2}$ correctly. Moreover, our scheme is additive in the sense that the distribution function for the sum of two quantities is the sum of the individual distribution functions: $\widehat{w}_{A+B}=\widehat{w}_{A}+\widehat{w}_{B}$. No such relation exists for the corresponding BLM scales. In particular, both $A$ and $B$ can have small BLM scales, but the BLM scale for the sum $(A+B)$ can be large, or vice versa. This brings us to the most important point, which is that the size of the BLM scale cannot always be used as an indicator for the rate of convergence of a perturbative series. The size of higher-order coefficients depends on the size of moments of the distribution function, not only on its central value, which determines the BLM scale.

\section{Asymptotic behaviour, infrared renormalons and the separation of long-distance contribu- tions}

Before we calculate the distribution function for specific examples we address the relevance of its asymptotic behaviour for large and small values of $\tau$. Clearly, the 
behaviour for $\tau \rightarrow \infty$ is related to the ultraviolet properties of the perturbative series. In particular, only if $\widehat{w}(\tau, z)$ vanishes faster than $1 / \tau$ the integral in (22) converges. Otherwise, our approach provides the framework for a consistent cutoff regularization of the series. Performing the integral up to a value $\tau_{\mathrm{UV}}$ corresponds to a hard momentum cutoff $\Lambda_{\mathrm{UV}}^{2}=\tau_{\mathrm{UV}} M^{2}$.

More subtle is the infrared region $\tau \rightarrow 0$. As long as one stays within perturbation theory one faces the problem that the integral over the distribution function runs over the Landau pole in the running coupling constant. One is forced to specify how to treat this pole, for instance by deforming the integration contour. In general, we may write

$$
\frac{\beta_{0} \alpha_{s}\left(\tau e^{C} M^{2}\right)}{4 \pi}=\frac{1}{\ln M^{2} / \Lambda_{V}^{2}+\ln \tau}=\mathrm{P}\left(\frac{1}{\ln \tau-\ln \tau_{L}}\right)+\eta \delta\left(\ln \tau-\ln \tau_{L}\right),
$$

where $\tau_{L}=\Lambda_{V}^{2} / M^{2}$ is the position of the Landau pole, "P" denotes the principle value, and $\eta$ is a complex parameter of order unity, which depends on the regularization prescription. This prescription dependence leads to an intrinsic ambiguity in the perturbative definition of $S\left(M^{2}, z\right)$, reflecting the fact that in (21) we are trying to sum up a series which is not Borel summable. This is how infrared renormalons make their appearance in our approach. Following Refs. [28]-[30], we define the renormalon ambiguity $\Delta S_{\text {ren }}$ in the value of $S\left(M^{2}, z\right)$ as the coefficient of $\eta$ and find

$$
\Delta S_{\mathrm{ren}}=\frac{\tau_{L}}{\beta_{0}} \widehat{w}\left(\tau_{L}, z\right) \simeq \frac{w_{0}(z)}{\beta_{0}}\left(\frac{\Lambda_{V}}{M}\right)^{2 k} .
$$

In the last step we have used the fact that $\tau_{L} \ll 1$ to expand the distribution function:

$$
\widehat{w}(\tau, z)=w_{0}(z) \tau^{k-1}+\ldots \text { for } \tau \rightarrow 0 .
$$

It is thus the asymptotic behaviour of the distribution function that determines the size of the renormalon ambiguity. Note that $k>0$ in order for the integral over the distribution function to be infrared convergent. We will see in examples that the power $k$ is related to the position of the nearest infrared renormalon pole in the Borel transform $\widehat{S}(u, z)$, which is located at $u_{\mathrm{IR}}=k$ in the Borel plane.

The appearance of infrared renormalons acts as a reminder that in (22) one is using perturbation theory in a regime where it is known to break down, namely in the infrared region. Hence, the result of any perturbative calculation in QCD is incomplete; it must be supplemented by non-perturbative corrections. Only the sum of all perturbative and non-perturbative contributions is unambiguous. Unlike any finite-order calculation, the representation (22) makes explicit that perturbative calculations contain long-distance contributions from the region of low momenta in Feynman diagrams. Moreover, it provides a convenient way to separate these long-distance contributions from the short-distance ones by introducing a hard factorization scale $\lambda$. Thus, our approach can be used to 
implement Wilson's construction of the operator product expansion (OPE) 31] in a literal way. Let us recall that the OPE is not designed to separate perturbative and non-perturbative effects, but to disentangle the physics on different length scales. In the calculation of short-distance corrections (the Wilson coefficient functions) one eliminates the contributions from small virtualities $(k<\lambda)$ in Feynman diagrams. These long-distance contributions are attributed to some matrix elements of higher-dimensional operators. Thus, we should write

$$
\begin{aligned}
S_{\mathrm{res}}\left(M^{2}, z\right) & =\int_{\lambda^{2} / M^{2}}^{\infty} \mathrm{d} \tau \widehat{w}(\tau, z) \frac{\alpha_{s}\left(\tau e^{C} M^{2}\right)}{4 \pi}+\int_{0}^{\lambda^{2} / M^{2}} \mathrm{~d} \tau \widehat{w}(\tau, z) \frac{\alpha_{s}\left(\tau e^{C} M^{2}\right)}{4 \pi} \\
& \equiv S_{\mathrm{sd}}\left(M^{2}, \lambda, z\right)+S_{\mathrm{ld}}\left(M^{2}, \lambda, z\right) .
\end{aligned}
$$

Note that the factorization point $\tau=\lambda^{2} / M^{2}$ corresponds to a scheme-dependent scale $\mu^{2}=e^{C} \lambda^{2}$ in the running coupling constant, with $\mu=\lambda$ in the $\mathrm{V}$ scheme. The value of $\alpha_{s}\left(e^{C} \lambda^{2}\right)$ is scheme-independent, however. As long as $\lambda$ is chosen large enough, the short-distance contribution $S_{\text {sd }}$ can be reliably calculated in perturbation theory and is free of renormalon ambiguities. The long-distance contribution $S_{\text {ld }}$ must be combined with other non-perturbative corrections. Only the sum of all long-distance contributions is well defined. Of course, the dependence on the arbitrary scale $\lambda$ must cancel in the final result. This $\lambda$-dependence can be controlled in perturbation theory by means of the renormalization-group equation

$$
\lambda \frac{\mathrm{d}}{\mathrm{d} \lambda} S_{\mathrm{sd}}\left(M^{2}, \lambda, z\right)=-\lambda \frac{\mathrm{d}}{\mathrm{d} \lambda} S_{\mathrm{ld}}\left(M^{2}, \lambda, z\right)=-\frac{\alpha_{s}\left(e^{C} \lambda^{2}\right)}{2 \pi} \frac{\lambda^{2}}{M^{2}} \widehat{w}\left(\lambda^{2} / M^{2}, z\right) .
$$

Since we have required that the quantity $S\left(M^{2}, z\right)$ be infrared safe, the longdistance contribution $S_{\mathrm{ld}}$ is finite, and it is usually assumed that it is small compared to the short-distance contribution. Eq. (30) allows us to quantify this statement. If the factorization scale is chosen such that $\lambda \ll M$, the $M$-dependence of the long-distance contribution is again determined by the asymptotic behaviour of the distribution function for small values of $\tau$. We find

$$
S_{\mathrm{ld}}\left(M^{2}, \lambda, z\right) \simeq \frac{w_{0}(z)}{M^{2 k}} \int_{0}^{\lambda^{2}} \mathrm{~d} \mu^{2} \mu^{2(k-1)} \frac{\alpha_{s}\left(e^{C} \mu^{2}\right)}{4 \pi} \equiv \pm\left(\frac{\Lambda(\lambda)}{M}\right)^{2 k}
$$

where $\Lambda(\lambda)$ is of order the QCD scale, and the sign is determined by the sign of $w_{0}$. The long-distance contribution is indeed parametrically small, suppressed by inverse powers of the large mass scale $M$. It is, of course, no accident that long-distance effects appear at the same order in $1 / M$ as infrared renormalon ambiguities. Both effects are exponentially small in the coupling constant and thus not seen in any finite-order perturbative calculation. Nevertheless, we will 
see that in cases where there is a nearby infrared renormalon (i.e. when the power $k$ is small) the long-distance contribution and the renormalon ambiguity can be numerically significant. One can try to estimate the size of $S_{\text {ld }}$ by incorporating certain non-perturbative effects into the integral over the distribution function, for instance by using a more realistic ansatz for the running coupling constant in the infrared region. For instance, one may assume that the coupling constant $\alpha_{s}\left(\mu^{2}\right)$ stays positive and approaches a constant for $\mu \rightarrow 0$. It is then possible to perform the $\tau$-integral without encountering a Landau pole. This yields to an unambiguous (though model-dependent) result for the long-distance contribution.

\section{Heavy quark systems}

We now illustrate the formalism developed above with some quantities related to heavy quarks, which provide prototype examples for large-scale problems in QCD. The large mass scale $M$ is provided by a heavy quark mass $m_{Q}$. Sects. 4.14.3 deal with the derivation of the distribution function for several quantities of interest. In Sect. 4.4 we present a numerical analysis of the results.

\subsection{Pole mass of a heavy quark}

We start with the relation between the pole mass $m_{Q}$ and the (infinite) bare mass $m_{0}$, which appears as a parameter in the QCD Lagrangian. We define

$$
S_{m}\left(m_{Q}^{2}\right)=\frac{m_{Q}}{m_{0}}-1
$$

and aim for a representation of this quantity as an integral over a distribution function $\widehat{w}_{m}(\tau)$, as shown in (22). In the large- $\beta_{0}$ limit, the Borel transform corresponding to the perturbative series for $S_{m}\left(m_{Q}^{2}\right)$ has the simple form [28]

$$
\widehat{S}_{m}(u)=6 C_{F}(1-u) \frac{\Gamma(u) \Gamma(1-2 u)}{\Gamma(3-u)},
$$

where $C_{F}=4 / 3$ is a colour factor. To derive the distribution function, we rewrite

$$
(1-u) \frac{\Gamma(u) \Gamma(1-2 u)}{\Gamma(3-u)}=\int_{0}^{1} \mathrm{~d} x \int_{0}^{1} \mathrm{~d} y x^{u-1}(1-x)^{-2 u} y^{1-u}
$$

and use the second relation in $(20)$ to obtain

$$
\widehat{w}_{m}(\tau)=6 C_{F} \int_{0}^{1} \frac{\mathrm{d} x}{x} \int_{0}^{1} \mathrm{~d} y y \delta\left(\tau-\frac{(1-x)^{2} y}{x}\right) .
$$


After a straightforward calculation, we find

$$
\widehat{w}_{m}(\tau)=C_{F}\left\{\frac{\tau}{2}+\left(1-\frac{\tau}{2}\right) \sqrt{1+\frac{4}{\tau}}\right\} .
$$

The small- $\tau$ behaviour of $\widehat{w}_{m}(\tau)$ is

$$
\widehat{w}_{m}(\tau)=\frac{2 C_{F}}{\sqrt{\tau}}+O(\sqrt{\tau})
$$

corresponding to $k=1 / 2$ in (29). This behaviour is associated with the infrared renormalon pole at $u=1 / 2$ in the Borel transform in (34). According to (28), the corresponding ambiguity in the perturbative series for $m_{Q} / m_{0}$ is

$$
\left(\Delta S_{m}\right)_{\mathrm{ren}}=\frac{\left(\Delta m_{Q}\right)_{\mathrm{ren}}}{m_{Q}}=\frac{8}{3 \beta_{0}} \frac{\Lambda_{V}}{m_{Q}}
$$

implying an ambiguity $\left(\Delta m_{Q}\right)_{\text {ren }}=\left(8 / 3 \beta_{0}\right) \Lambda_{V}$ in the value of the pole mass [28, 29].

For large values of $\tau$ the distribution function decreases as $3 C_{F} / \tau$, so that the integral in (22) is logarithmically divergent. The divergence is removed by a renormalization of the bare mass. It turns out that the calculation of the distribution function is complicated if one chooses the $\overline{\mathrm{MS}}$ scheme for this purpose. The Borel transform corresponding to the ratio $m_{Q} / \bar{m}_{Q}\left(m_{Q}\right)$ is given by [28]

$$
\widehat{S}_{m}^{\overline{\mathrm{MS}}}(u)=C_{F}\left\{6(1-u) \frac{\Gamma(u) \Gamma(1-2 u)}{\Gamma(3-u)}+e^{-5 u / 3}\left(-\frac{3}{u}+R(u)\right)\right\},
$$

where

$$
R(u)=-\frac{5}{2}+\frac{35}{24} u+\left(\zeta(3)-\frac{83}{144}\right) u^{2}+\ldots
$$

is a rather complicated function. Instead, we find it instructive to consider a class of renormalization schemes $\mathrm{R}[r]$ which are more convenient for our calculation. We define the Borel transform corresponding to the ratio $m_{Q} / m_{Q}^{\mathrm{R}}$ as

$$
\widehat{S}_{m}^{\mathrm{R}}(u)=C_{F}\left\{6(1-u) \frac{\Gamma(u) \Gamma(1-2 u)}{\Gamma(3-u)}-\frac{3}{u} e^{-r u}\right\}
$$

and treat $r$ as a free parameter. From a comparison of the Borel transforms $\widehat{S_{m}^{\mathrm{MS}}}$ and $\widehat{S}_{m}^{\mathrm{R}}$ one finds that the relation between the so-defined mass $m_{Q}^{\mathrm{R}}$ and the mass renormalized in the $\overline{\mathrm{MS}}$ scheme is given by

$$
\begin{aligned}
\frac{m_{Q}^{\mathrm{R}}}{\bar{m}_{Q}\left(m_{Q}\right)}= & -\left(r-\frac{5}{6}\right) \frac{\bar{\alpha}_{s}}{\pi}+\frac{\beta_{0}}{8}\left(r^{2}-\frac{10 r}{3}+\frac{15}{4}\right)\left(\frac{\bar{\alpha}_{s}}{\pi}\right)^{2} \\
& -\frac{\beta_{0}^{2}}{48}\left(r^{3}-5 r^{2}+\frac{25 r}{3}-\frac{751}{216}-2 \zeta(3)\right)\left(\frac{\bar{\alpha}_{s}}{\pi}\right)^{3}+\ldots,
\end{aligned}
$$


where $\bar{\alpha}_{s}=\alpha_{s}\left(m_{Q}^{2}\right)$ denotes the coupling constant in the $\overline{\mathrm{MS}}$ scheme. It is a simple exercise to calculate the distribution function for the ratio $m_{Q} / m_{Q}^{\mathrm{R}}$. The result is

$$
\widehat{w}_{m}^{\mathrm{R}}(\tau)=\widehat{w}_{m}(\tau)-\frac{3 C_{F}}{\tau} \Theta\left(\tau-e^{r}\right),
$$

which simply amounts to a subtraction of the high-momentum contributions, leaving the low-momentum region unaffected. The subtracted distribution function falls off as $1 / \tau^{2}$ for large values of $\tau$, so that the integral over $\tau$ is convergent.

With the distribution function $\widehat{w}_{m}^{\mathrm{R}}(\tau)$ we compute

$$
\begin{aligned}
N & =\left(\frac{3}{8}+\frac{3 r}{4}\right) C_{F}=\frac{1}{2}+r, \\
\langle\ln \tau\rangle & =\frac{r^{2}-\frac{1}{2}-\frac{2}{3} \pi^{2}}{2 r+1}, \\
\left\langle\ln ^{2} \tau\right\rangle & =\frac{2}{3} \frac{r^{3}+\frac{3}{4}+12 \zeta(3)+\pi^{2}}{2 r+1},
\end{aligned}
$$

which is all one needs to calculate the BLM scale and the parameter $\Delta$ defined in (23). Two cases are particularly interesting:

$$
\begin{array}{ll}
\text { scheme R1: } & r=\frac{5}{6}, \\
\text { scheme R2: } & r=r_{0}=\sqrt{\frac{1}{2}+\frac{2 \pi^{2}}{3}} \simeq 2.661 .
\end{array}
$$

Since the one-loop coefficient in (43) vanishes for $r=5 / 6$, scheme $\mathrm{R} 1$ is similar to the $\overline{\mathrm{MS}}$ scheme. The relation between the mass definitions in the two schemes is

$$
\frac{m_{Q}^{\mathrm{R} 1}}{\bar{m}_{Q}\left(m_{Q}\right)}=1+0.208 \beta_{0}\left(\frac{\bar{\alpha}_{s}}{\pi}\right)^{2}+0.038 \beta_{0}^{2}\left(\frac{\bar{\alpha}_{s}}{\pi}\right)^{3}+\ldots,
$$

which is a nicely converging series. For the bottom quark the ratio equals 1.009. The scheme R2 is chosen such that $\langle\ln \tau\rangle=0$, so that the BLM scale is given by $\mu_{\mathrm{BLM}}^{2}=e^{C} m_{Q}^{2}$. In this case one finds

$$
\frac{m_{Q}^{\mathrm{R} 2}}{\bar{m}_{Q}\left(m_{Q}\right)}=1-1.827 \frac{\bar{\alpha}_{s}}{\pi}+0.245 \beta_{0}\left(\frac{\bar{\alpha}_{s}}{\pi}\right)^{2}+0.006 \beta_{0}^{2}\left(\frac{\bar{\alpha}_{s}}{\pi}\right)^{3}+\ldots
$$

which has a sizeable one-loop coefficient but is again nicely converging. For the bottom quark the ratio equals 0.883 .

In Table 1 we give, for the case of the bottom quark, the results for the BLM scale in three different mass renormalization schemes (R1, R2, $\overline{\mathrm{MS}}$ ) and for two renormalization schemes for the coupling constant (V and $\overline{\mathrm{MS}}$ ). We also quote the corresponding scheme-independent values of $\alpha_{s}\left(\mu_{\mathrm{BLM}}^{2}\right)$, as well as the parameters 
Table 1: Parameters related to the distribution function $\widehat{w}_{m}^{\mathrm{R}}(\tau)$ for three different mass renormalization schemes. We use the one-loop expression for the running coupling constant in the $\overline{\mathrm{MS}}$ scheme, normalized such that $\alpha_{s}\left(m_{b}^{2}\right)=0.218$ for $m_{b}=4.8 \mathrm{GeV}$.

\begin{tabular}{l|ccccr}
\hline \hline & $\mu_{\mathrm{BLM}}^{V}$ & $\mu_{\mathrm{BLM}}^{\overline{\mathrm{MS}}}$ & $\alpha_{s}\left(\mu_{\mathrm{BLM}}^{2}\right)$ & $\Delta$ & $\delta_{\mathrm{BLM}}$ \\
\hline$m_{b} / m_{b}^{\mathrm{R} 1}$ & $0.302 m_{b}$ & $0.131 m_{b}$ & 0.55 & 0.672 & 0.10 \\
$m_{b} / m_{b}^{\mathrm{R} 2}$ & $m_{b}$ & $0.435 m_{b}$ & 0.29 & 4.628 & 0.17 \\
$m_{b} / \bar{m}_{b}\left(m_{b}\right)$ & $0.221 m_{b}$ & $0.096 m_{b}$ & 0.73 & -4.337 & -1.19 \\
\hline \hline
\end{tabular}

$\Delta$ and $\delta_{\mathrm{BLM}}$ defined in (23) and (25). We note that the distribution function corresponding to the $\overline{\mathrm{MS}}$ scheme must satisfy

$$
\begin{aligned}
N & =C_{F}=\frac{4}{3} \\
\langle\ln \tau\rangle & =-\frac{53}{96}-\frac{\pi^{2}}{4} \simeq-3.019 \\
\left\langle\ln ^{2} \tau\right\rangle & =\frac{7}{2} \zeta(3)-\frac{1637}{864}+\frac{\pi^{2}}{4} \simeq 4.780,
\end{aligned}
$$

as can be derived from an expansion of the Borel transform (40) in powers of $u$. The large negative values of the parameters $\Delta$ and $\delta_{\text {BLM }}$ for the ratio $m_{b} / \bar{m}_{b}\left(m_{b}\right)$ indicate that there are large corrections to the BLM scheme which decrease the value of the perturbative series. In other words, the BLM scale is too low and does not really represent an "average" virtuality. The reason for the strong dependence of the BLM scale on the subtraction scheme becomes apparent from the shape of the distribution functions shown in Fig. 1. We find it most useful to show the product $\tau \widehat{w}(\tau)$ as a function of $\ln \tau$, since then the integrals $\left\langle\ln ^{n} \tau\right\rangle$ have a direct graphical interpretation. The long arrows indicate the position of the BLM scale in the schemes R1 and R2. The small arrow shows the point $\tau=\lambda^{2} / m_{b}^{2}$ for $\lambda=1$ $\mathrm{GeV}$, which will later be used to separate short- and long-distance contributions [cf. (30)]. In order to associate mass scales with the $\tau$-values in the figure, we note that

$$
\ln \frac{\mu^{2}}{m_{b}^{2}}=\ln \tau+C,
$$

where $\mu$ is the scale in the running coupling constant. In the $\mathrm{V}$ scheme, where $C=0$, the point $\tau=\lambda^{2} / m_{b}^{2}$ corresponds to the scale $\mu=\lambda$, whereas $\ln \tau=0$ corresponds to $\mu=m_{b}$. In the $\overline{\mathrm{MS}}$ scheme, where $C=-5 / 3$, the point $\mu=m_{b}$ corresponds to $\ln \tau=5 / 3$.

We observe that the pole mass gets contributions from all momentum scales, 


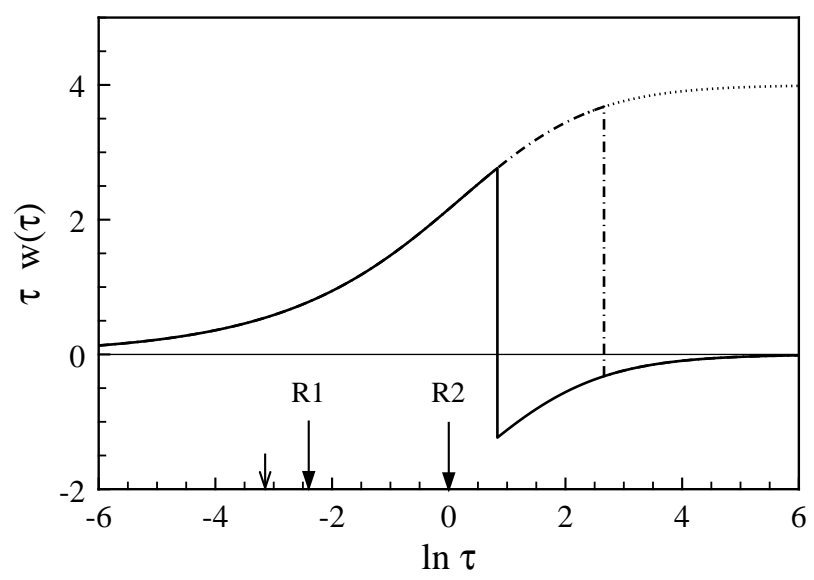

Figure 1: Distribution function for the ratio $m_{b} / m_{b}^{\mathrm{R}}$ in the two renormalization schemes R1 (solid line) and R2 (dashed-dotted line). The dotted line shows the unsubtracted distribution function for the ratio of the pole mass and the bare mass. The long arrows indicate the position of the BLM scale in the schemes R1 and R2. The small arrow shows the factorization point, which separates short- and long-distance contributions.

and it is only the subtraction of the high-momentum tail that leads to a negative value of $\langle\ln \tau\rangle$ in the scheme $\mathrm{R} 1$ (and similar for the $\overline{\mathrm{MS}}$ scheme). If the subtraction point is chosen as low as in R1, significant cancellations take place between positive and negative contributions in the integral over the distribution function. The results is a small one-loop coefficient $N$ in (23), yielding in turn a small BLM scale. Thus, the interpretation of the BLM scale as a "typical" scale in a process becomes misleading if the distribution function gets contributions of opposite sign. Note that the situation encountered here is generic for quantities which require a subtraction of ultraviolet divergences. In such cases, a low value of the BLM scale does not necessarily imply a bad convergence of the perturbative series.

The series in (43) is interesting by itself. Since both $m_{Q}^{\mathrm{R}}$ and $\bar{m}_{Q}\left(m_{Q}\right)$ are subtracted at a large mass scale, their ratio has a well-behaved expansion in powers of $\alpha_{s}\left(m_{Q}^{2}\right)$ as long as $r$ is of order unity. In fact, for the two choices of $r$ discussed above the series was rapidly converging. Nevertheless, the BLM scale corresponding to this series exhibits a strong dependence on $r$. We find $\mu_{\mathrm{BLM}}^{2}=e^{C}\left[\gamma(r) m_{Q}\right]^{2}$ with

$$
\ln \gamma(r)=\frac{r^{2}+\frac{35}{36}}{4 r-\frac{10}{3}} .
$$

This function is shown in Fig. 2. The reason for the dramatic scheme dependence 


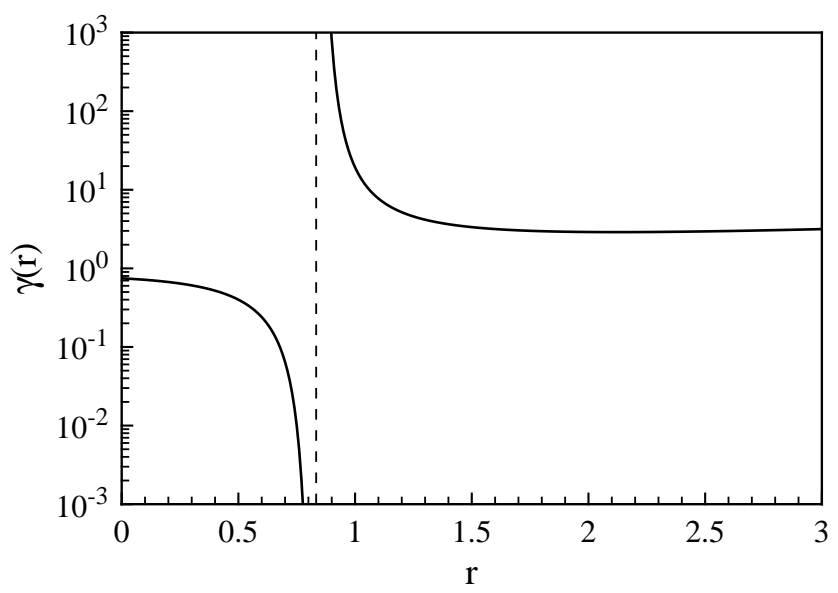

Figure 2: The function $\gamma(r)$ defined in (51).

of $\mu_{\mathrm{BLM}}$ is simply that the one-loop coefficient vanishes for $r=5 / 6$. This example shows that a small BLM scale is not always related to a badly converging series. It also shows the disadvantage of non-additivity of the BLM prescription: As shown in Table 1, the BLM scale for the ratio $m_{Q} / \bar{m}_{Q}\left(m_{Q}\right)$ is rather low. However, the same ratio can be obtained by combining the two series for $m_{Q} / m_{Q}^{\mathrm{R}}$ and $m_{Q}^{\mathrm{R}} / \bar{m}_{Q}\left(m_{Q}\right)$ in the scheme R2, both of which have a much larger BLM scale. On the other hand, calculating $m_{Q} / \bar{m}_{Q}\left(m_{Q}\right)$ by combining integrals over the appropriate distribution functions one obtains a unique result, in which the contributions from all scales are properly taken into account.

\subsection{Matching coefficients of heavy-light currents}

A convenient way to analyse hadronic matrix elements of the flavour-changing weak current $j^{\mu}=\bar{q} \gamma^{\mu}\left(1-\gamma_{5}\right) Q$ between a hadron containing the heavy quark $Q$ and some light final state is to go over to an effective theory, the so-called heavy quark effective theory [32, in which such matrix elements are systematically expanded in powers of $1 / m_{Q}$. When QCD is matched onto the effective theory, the current gets replaced by 33

$$
j^{\mu} \rightarrow C_{1}\left(m_{Q}^{2}\right) \bar{q} \gamma^{\mu}\left(1-\gamma_{5}\right) h_{v}+C_{2}\left(m_{Q}^{2}\right) \bar{q} v^{\mu}\left(1+\gamma_{5}\right) h_{v}+O\left(1 / m_{Q}\right),
$$

where $v$ is the four-velocity of the hadron that contains the heavy quark, and $h_{v}$ is the velocity-dependent heavy quark field in the effective theory. The above form of the currents is correct if one uses a regularization scheme with anti-commuting $\gamma_{5}$. The matching coefficients $C_{i}\left(m_{Q}^{2}\right)$ can be calculated in perturbation theory by comparing quark matrix elements of the currents in QCD and in the effective theory. We define

$$
S_{1}\left(m_{Q}^{2}\right)=C_{1}\left(m_{Q}^{2}\right)-1, \quad S_{2}\left(m_{Q}^{2}\right)=C_{2}\left(m_{Q}^{2}\right) .
$$


Table 2: Parameters related to the distribution function $\widehat{w}_{2}(\tau)$, which is relevant for the ratio of heavy meson decay constants.

\begin{tabular}{c|cccccc}
\hline \hline & $\mu_{\mathrm{BLM}}^{V}$ & $\mu_{\mathrm{BLM}}^{\overline{\mathrm{MS}}}$ & $\alpha_{s}\left(\mu_{\mathrm{BLM}}^{2}\right)$ & $\sigma_{\tau}$ & $\Delta$ & $\delta_{\mathrm{BLM}}$ \\
\hline$f_{B}^{\text {stat }} / f_{B^{*}}^{\text {stat }}$ & $0.472 m_{b}$ & $0.205 m_{b}$ & 0.41 & 2.798 & 7.830 & 0.67 \\
\hline \hline
\end{tabular}

In the large- $\beta_{0}$ limit, the Borel transforms of the perturbative series for these quantities are given by 30

$$
\begin{aligned}
& \widehat{S}_{1}(u)=C_{F}\left(3 u^{2}-u-3\right) \frac{\Gamma(u) \Gamma(1-2 u)}{\Gamma(3-u)}, \\
& \widehat{S}_{2}(u)=4 C_{F} \frac{\Gamma(1+u) \Gamma(1-2 u)}{\Gamma(3-u)} .
\end{aligned}
$$

The corresponding distribution functions can be calculated as outlined in the previous section. We find

$$
\begin{aligned}
& \widehat{w}_{1}(\tau)=\frac{C_{F}}{2}\left\{1-\frac{7}{6} \tau-\frac{3}{2 \sqrt{1+4 / \tau}}-\frac{11-7 \tau}{6} \sqrt{1+\frac{4}{\tau}}\right\}, \\
& \widehat{w}_{2}(\tau)=\frac{2 C_{F}}{3}\left\{(1+\tau) \sqrt{1+\frac{4}{\tau}}-3-\tau\right\} .
\end{aligned}
$$

The asymptotic behaviour for small values of $\tau$ is

$$
\widehat{w}_{1}(\tau)=-\frac{11 C_{F}}{6 \sqrt{\tau}}+O(1), \quad \widehat{w}_{2}(\tau)=\frac{4 C_{F}}{3 \sqrt{\tau}}+O(1),
$$

corresponding to the infrared renormalon poles at $u=1 / 2$ in the Borel transforms in (54). If we relate the corresponding renormalon ambiguities to the ambiguity in the value of the pole mass [cf. (38) and (39)], we recover the relations

$$
\left(\Delta C_{1}\right)_{\mathrm{ren}}=-\frac{11}{12} \frac{\left(\Delta m_{Q}\right)_{\mathrm{ren}}}{m_{Q}}, \quad\left(\Delta C_{2}\right)_{\mathrm{ren}}=\frac{2}{3} \frac{\left(\Delta m_{Q}\right)_{\mathrm{ren}}}{m_{Q}}
$$

derived in Ref. 30]. The behaviour of the distribution functions for large values of $\tau$ is $\widehat{w}_{1}(\tau) \sim 1 / \tau$ and $\widehat{w}_{2}(\tau) \sim 1 / \tau^{2}$. The slow fall-off of $\widehat{w}_{1}(\tau)$ leads to a logarithmic divergence in $C_{1}\left(m_{Q}^{2}\right)$, which must be removed by renormalization.

As an application of these results, consider the ratio of the decay constants of the pseudoscalar and vector mesons $B$ and $B^{*}$ in the so-called static limit, where terms of order $\Lambda_{\mathrm{QCD}} / m_{b}$ are neglected on the level of hadronic matrix elements. 
In this limit, one finds 34

$$
\frac{f_{B}^{\text {stat }}}{f_{B^{*}}^{\text {stat }}}=1+\frac{C_{2}\left(m_{Q}^{2}\right)}{C_{1}\left(m_{Q}^{2}\right)}=1+\int_{0}^{\infty} \mathrm{d} \tau \widehat{w}_{2}(\tau) \frac{\alpha_{s}\left(\tau e^{C} m_{b}^{2}\right)}{4 \pi}+\ldots
$$

where the ellipses represent terms not resummed in our approach. Since $C_{1}\left(m_{Q}^{2}\right)=$ $1+O\left(1 / \beta_{0}\right)$, the distribution function is the same as for the matching coefficient $C_{2}\left(m_{Q}^{2}\right)$.

For the distribution function $\widehat{w}_{2}(\tau)$ we compute

$$
\begin{aligned}
N & =\frac{C_{F}}{2}=\frac{2}{3}, \\
\langle\ln \tau\rangle & =-\frac{3}{2}, \\
\left\langle\ln ^{2} \tau\right\rangle & =\frac{7}{2}+\frac{2 \pi^{2}}{3} .
\end{aligned}
$$

The resulting value of the BLM scale and some other parameters are summarized in Table 2. Since in this case the distribution function is positive definite, the parameter $\sigma_{\tau}=\sqrt{\Delta}$ corresponds to the width of the distribution function. The width is very large, about three units in $\ln \tau$. This is clearly reflected in the shape of the function $\tau \widehat{w}_{2}(\tau)$ shown in Fig. 3. As indicated by the position of the arrows, the BLM scale is rather low, close to the factorization point; the distribution is broad and extends well into the infrared region. As a consequence, the convergence of the series is bad, as reflected in the large value of the parameter $\delta_{\mathrm{BLM}}$ in Table 2 .

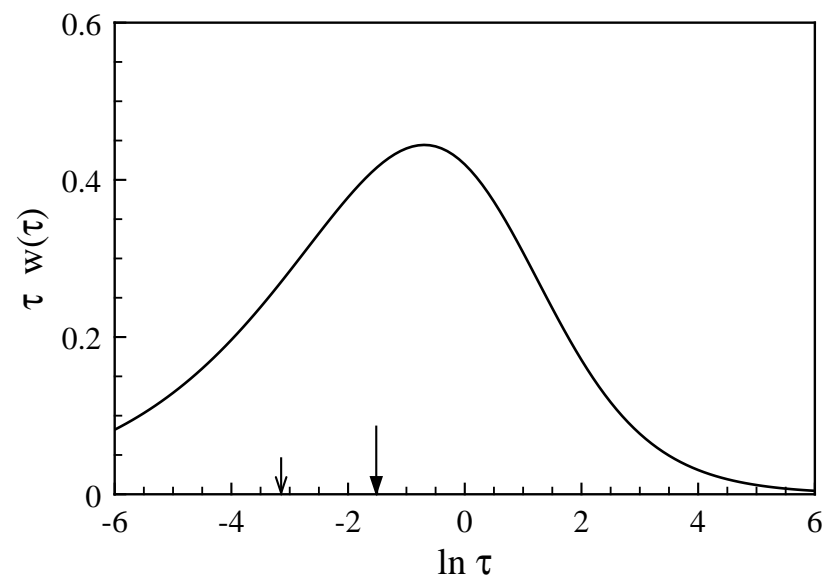

Figure 3: Distribution function for the ratio $f_{B}^{\text {stat }} / f_{B^{*}}^{\text {stat }}$. The right arrow indicates the BLM scale, the left one the factorization point.

In the case of the ratio $m_{Q} / m_{Q}^{\mathrm{R}}$ considered above, the BLM scale was low since the high-momentum contributions were removed, in an ad hoc way, by the 
renormalization of the bare quark mass. On the contrary, in the present case there is a physical reason why the "typical" momenta in the one-loop calculation are much lower than the "natural" scale $m_{b}$. The flavour-changing vector and axial vector currents are partially conserved, implying that they do not receive radiative corrections from scales much above the masses of their component fields [35]. Roughly speaking, then, the matching coefficients receive contributions from scales $0<\mu<m_{Q}$, which is in fact the behaviour reflected in Fig. 3. (Recall that $\mu=m_{Q}$ corresponds to $\ln \tau=0$ in the $\mathrm{V}$ scheme, and $\ln \tau=5 / 3$ in the $\overline{\mathrm{MS}}$ scheme.) How fast the distribution falls off in the infrared region is determined by the location of the nearest infrared renormalon, which determines the low$\tau$ behaviour of the distribution function. Since in the present case the nearest renormalon is located at $u=1 / 2$ in the Borel plane, i.e. at the smallest possible value of $u$, the fall-off is the slowest possible one, and thus there are substantial contributions from the low-momentum region.

\subsection{Matching coefficients of heavy-heavy currents}

Let us now consider a two-scale problem, namely the matching of flavour-changing currents containing two heavy quarks onto their counterparts in the heavy quark effective theory. At the so-called zero recoil point, where the two heavy quarks move at the same velocity, one finds [33]

$$
\begin{gathered}
\bar{c} \gamma^{\mu} b \rightarrow \eta_{V} \bar{h}_{v}^{c} \gamma^{\mu} h_{v}^{b}+O\left(1 / m_{Q}^{2}\right), \\
\bar{c} \gamma^{\mu} \gamma_{5} b \rightarrow \eta_{A} \bar{h}_{v}^{c} \gamma^{\mu} \gamma_{5} h_{v}^{b}+O\left(1 / m_{Q}^{2}\right) .
\end{gathered}
$$

The coefficients $\eta_{V}$ and $\eta_{A}$ take into account finite renormalization effects. The coefficient $\eta_{A}$ of the axial vector current plays a crucial role in the model-independent determination of $\left|V_{c b}\right|$ from $B \rightarrow D^{*} \ell \bar{\nu}_{\ell}$ decays [36].

We choose $M=\sqrt{m_{b} m_{c}}$ as the "natural" mass scale and define the ratio $z=m_{c} / m_{b}$. It is convenient to study the quantities

$$
S_{V}\left(m_{b} m_{c}, z\right)=\eta_{V}-1, \quad S_{V-A}\left(m_{b} m_{c}, z\right)=\eta_{V}-\eta_{A},
$$

since the structure of the perturbative corrections is very similar for $\eta_{V}$ and $\eta_{A}$. The corresponding Borel transforms in the large- $\beta_{0}$ limit are 30, 37

$$
\begin{aligned}
\widehat{S}_{V}(u, z)= & C_{F} \frac{\Gamma(u) \Gamma(1-2 u)}{\Gamma(2-u)}\left\{\frac{2(1+u)}{2-u} \frac{z^{u}-z^{1-u}}{1-z}+\frac{2(1-u)}{1+2 u} \frac{z^{-u}-z^{1+u}}{1-z}\right. \\
& \left.-\frac{1+z}{1-z}\left(z^{u}-z^{-u}\right)-\frac{3\left(1-u^{2}\right)}{2-u}\left(z^{u}+z^{-u}\right)\right\} \\
\widehat{S}_{V-A}(u, z)= & 4 C_{F} \frac{\Gamma(1+u) \Gamma(1-2 u)}{\Gamma(3-u)} \frac{z^{u}-z^{1-u}}{1-z} .
\end{aligned}
$$


After a straightforward calculation, we obtain the distribution functions

$$
\begin{aligned}
\widehat{w}_{V}(\tau, z)=C_{F} & \left\{\frac{1-\tau}{2} \frac{(1-z)^{2}}{z}-\frac{3}{4} \tau \frac{\left(1-z^{2}\right)^{2}}{z^{2}}\right. \\
& -\frac{3}{4} \frac{z}{\sqrt{1+4 /(\tau z)}}-\frac{3}{4} \frac{1}{z \sqrt{1+4 z / \tau}} \\
+ & \sqrt{1+\frac{4}{\tau z}}\left[-\frac{z}{4}+\frac{3}{4} \tau z^{2}-\frac{2}{\tau} \frac{z}{1-z}+\frac{\tau}{2} \frac{z^{2}}{1-z}\right] \\
+ & \left.\sqrt{1+\frac{4 z}{\tau}}\left[-\frac{1}{4 z}+\frac{3}{4} \frac{\tau}{z^{2}}+\frac{2}{\tau} \frac{1}{1-z}-\frac{\tau}{2} \frac{1}{z(1-z)}\right]\right\}, \\
\widehat{w}_{V-A}(\tau, z)=2 C_{F} & \left\{1+\tau \frac{1+z+z^{2}}{3 z}\right. \\
& \left.-\frac{1}{3(1-z)}\left[\left(1+\frac{\tau}{2}\right) \sqrt{1+\frac{4 z}{\tau}}-z(1+\tau z) \sqrt{1+\frac{4}{\tau z}}\right]\right\} .
\end{aligned}
$$

The asymptotic behaviour for small values of $\tau$ is

$$
\begin{aligned}
\widehat{w}_{V}(\tau, z) & =-C_{F} \frac{(1-z)^{2}}{2 z}+O(\sqrt{\tau}), \\
\widehat{w}_{V-A}(\tau, z) & =2 C_{F}+O(\sqrt{\tau}) .
\end{aligned}
$$

It is associated with infrared renormalon poles at $u=1$ in the Borel plane. In fact, a careful investigation of (62) shows that there are no poles at $u=1 / 2$. The corresponding renormalon ambiguities in the matching coefficients are

$$
\begin{aligned}
& \left(\Delta \eta_{V}\right)_{\mathrm{ren}}=-\frac{2}{3 \beta_{0}}\left(\frac{\Lambda_{V}}{m_{c}}-\frac{\Lambda_{V}}{m_{b}}\right)^{2}, \\
& \left(\Delta \eta_{A}\right)_{\mathrm{ren}}=-\frac{2}{3 \beta_{0}}\left(\frac{\Lambda_{V}}{m_{c}}+\frac{\Lambda_{V}}{m_{b}}\right)^{2} .
\end{aligned}
$$

This agrees with the results obtained in Ref. [30. For large values of $\tau$, both distribution functions fall off proportional to $1 / \tau^{2}$.

With the above distribution functions, we compute

$$
\begin{aligned}
N & =\frac{C_{F}}{4} \phi(z) \simeq 0.236 \\
\langle\ln \tau\rangle & =\frac{3}{2} \\
\left\langle\ln ^{2} \tau\right\rangle & =\frac{2 \pi^{2}}{3}+\frac{9}{2}+\frac{1}{3} \ln ^{2} z-4 \frac{\ln ^{2} z}{\phi(z)} \simeq 3.372,
\end{aligned}
$$


Table 3: Parameters obtained from the distribution functions for the matching coefficients $\eta_{V}$ and $\left(\eta_{V}-\eta_{A}\right)$ of heavy quark currents. We use $m_{b}=4.8 \mathrm{GeV}$ and $m_{c}=1.44 \mathrm{GeV}$.

\begin{tabular}{l|cccccc}
\hline \hline & $\mu_{\mathrm{BLM}}^{V}$ & $\mu_{\mathrm{BLM}}^{\overline{\mathrm{MS}}}$ & $\alpha_{s}\left(\mu_{\mathrm{BLM}}^{2}\right)$ & $\sigma_{\tau}$ & $\Delta$ & $\delta_{\mathrm{BLM}}$ \\
\hline$\eta_{V}$ & $2.117 \sqrt{m_{b} m_{c}}$ & $0.920 \sqrt{m_{b} m_{c}}$ & 0.27 & 1.059 & 1.121 & 0.04 \\
$\eta_{V}-\eta_{A}$ & $1.445 \sqrt{m_{b} m_{c}}$ & $0.628 \sqrt{m_{b} m_{c}}$ & 0.31 & 2.069 & 4.281 & 0.19 \\
\hline \hline
\end{tabular}

for $\widehat{w}_{V}(\tau, z)$, and

$$
\begin{aligned}
N & =\frac{C_{F}}{2}=\frac{2}{3} \\
\langle\ln \tau\rangle & =\frac{1}{2}+\frac{1}{3} \phi(z) \simeq 0.736 \\
\left\langle\ln ^{2} \tau\right\rangle & =\frac{2 \pi^{2}}{3}-\frac{5}{2}+\ln ^{2} z-\phi(z) \simeq 4.821
\end{aligned}
$$

for $\widehat{w}_{V-A}(\tau, z)$, where

$$
\phi(z)=-3 \frac{1+z}{1-z} \ln z-6=\frac{\ln ^{2} z}{2}-\frac{\ln ^{4} z}{120}+O\left(\ln ^{6} z\right) .
$$

We have used the value $z=m_{c} / m_{b}=0.3$ in the numerical analysis. The corresponding BLM scales and the values of the parameters $\sigma_{\tau}, \Delta$ and $\delta_{\mathrm{BLM}}$ are given in Table 3. The distribution functions are shown in Fig. 4 . In both cases the BLM scales are comfortably large and are clearly separated from the factorization point, which corresponds to $\tau=\lambda^{2} / m_{b} m_{c}$ with $\lambda=1 \mathrm{GeV}$. We note that the scales $\mu=m_{b}$ and $\mu=m_{c}$ correspond to $\ln \tau= \pm 1.204-C$, with $C=0$ in the $\mathrm{V}$ scheme and $C=-5 / 3$ in the $\overline{\mathrm{MS}}$ scheme. The distributions fall off rapidly in the infrared region. Therefore, both series converge much better than in the case of the heavy-light current considered in the previous section.

The physical reason for this behaviour is again related to current conservation. As before, the currents are conserved in the ultraviolet region, i.e. for scales $\mu \gg m_{b}$. But in contrast to heavy-light currents, currents containing two heavy quarks moving at the same velocity are also conserved in the infrared region, i.e. for $\mu \ll m_{c}$. In fact, at zero recoil the anomalous dimension associated with such currents in the heavy quark effective theory vanishes to all orders in perturbation theory [38, 39]. This means that the matching coefficients receive sizeable contributions only from scales $m_{c}<\mu<m_{b}$, which is in accordance with the behaviour exhibited in Fig. 4 . 


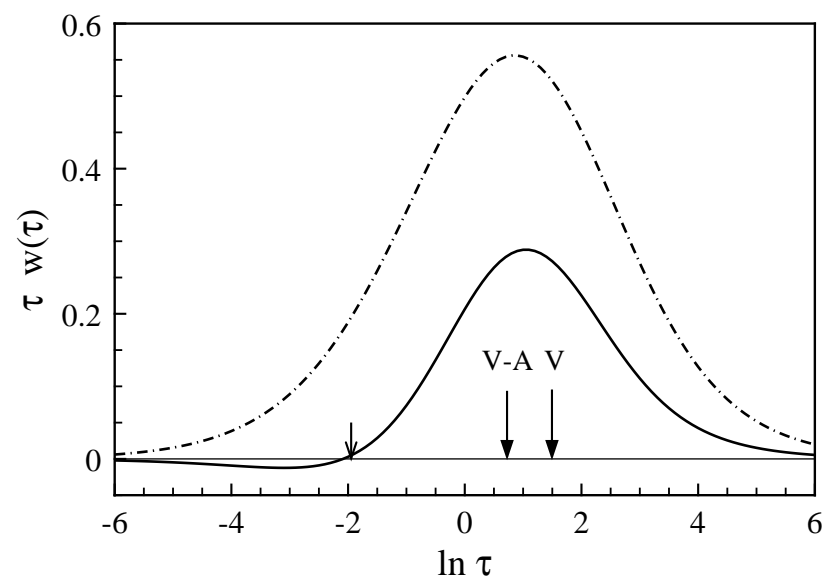

Figure 4: Distribution functions for $\eta_{V}$ (solid line) and $\left(\eta_{V}-\eta_{A}\right)$ (dasheddotted line). The long arrows show the BLM scales, the short arrow indicates the factorization point.

\subsection{Numerical analysis}

Let us now analyse our results. For each of the quantities $S\left(M^{2}\right)$ considered in the previous sections, we compare the following approximations: the oneand (partial) two-loop expressions evaluated using the "natural" scale $M$ in the running coupling constant, the one-loop expression evaluated using the BLM scale, the truncated series including the first correction to the BLM scheme given by the term proportional to $\Delta$ in (22), the partial resummation provided by the integral over the distribution function. In the latter case we use the principle value prescription to regularize the Landau pole in the running coupling constant. We define

$$
\begin{aligned}
S_{1-\text { loop }}\left(M^{2}\right) & =N \frac{\alpha_{s}\left(M^{2}\right)}{\pi}, \\
S_{2-\text { loop }}\left(M^{2}\right) & =N \frac{\alpha_{s}\left(M^{2}\right)}{\pi}\left\{1-(C+\langle\ln \tau\rangle) \frac{\beta_{0} \alpha_{s}\left(M^{2}\right)}{4 \pi}\right\}, \\
S_{\mathrm{BLM}}\left(M^{2}\right) & =N \frac{\alpha_{s}\left(\mu_{\mathrm{BLM}}^{2}\right)}{\pi}, \\
S_{\mathrm{BLM}}\left(M^{2}\right) & =N \frac{\alpha_{s}\left(\mu_{\mathrm{BLM}}^{2}\right)}{\pi}\left\{1+\Delta\left(\frac{\beta_{0} \alpha_{s}\left(\mu_{\mathrm{BLM}}^{2}\right)}{4 \pi}\right)^{2}\right\}, \\
S_{\mathrm{res}}\left(M^{2}\right) & =N\left\langle\frac{\alpha_{s}\left(\tau e^{C} M^{2}\right)}{\pi}\right\rangle .
\end{aligned}
$$

Note that $S_{2-\text { loop }}$ takes into account only part of the two-loop corrections, namely those proportional to $\beta_{0} \alpha_{s}^{2}$. As pointed out in the introduction, for the quantities considered here it is known that the remaining two-loop corrections are very 
Table 4: Comparison of various approximations for the quantities shown in the first column.

\begin{tabular}{l|cccccc}
\hline \hline & $S_{1-\text { loop }}$ & $S_{2-\text { loop }}$ & $S_{\text {BLM }}$ & $S_{\text {BLM }}$ & $S_{\text {res }}$ & $\Delta S_{\text {ren }}$ \\
\hline$m_{b} / m_{b}^{\mathrm{R} 1}$ & 0.092 & 0.146 & 0.234 & 0.259 & 0.178 & 0.025 \\
$m_{b} / m_{b}^{\mathrm{R} 2}$ & 0.219 & 0.271 & 0.288 & 0.336 & 0.304 & 0.025 \\
$m_{b} / \bar{m}_{b}\left(m_{b}\right)$ & 0.092 & 0.155 & 0.310 & -0.059 & 0.188 & 0.025 \\
$f_{B}^{\text {stat }} / f_{B^{*}}^{\text {stat }}$ & 0.046 & 0.067 & 0.086 & 0.144 & 0.076 & 0.017 \\
$\eta_{V}$ & 0.020 & 0.020 & 0.020 & 0.021 & 0.023 & -0.003 \\
$\eta_{V}-\eta_{A}$ & 0.056 & 0.065 & 0.067 & 0.079 & 0.081 & 0.007 \\
\hline \hline
\end{tabular}

small. We use the one-loop expression for the running coupling constant in the $\overline{\mathrm{MS}}$ scheme with $\Lambda_{5}=111 \mathrm{MeV}, \Lambda_{4}=150 \mathrm{MeV}$ and $\Lambda_{3}=177 \mathrm{MeV}$, so that the coupling constant is continuous when one crosses the quark thresholds at $m_{b}=4.8$ $\mathrm{GeV}$ and $m_{c}=1.44 \mathrm{GeV}$. For reference purposes we quote that $\alpha_{s}\left(m_{b}^{2}\right) \simeq 0.218$ and $\alpha_{s}\left(m_{c}^{2}\right) \simeq 0.333$. Our results are shown in Table $甘$. In the last column we give the value of the renormalon ambiguity defined in (28). To obtain it we use $\beta_{0}=9$ and $\Lambda_{V}=408 \mathrm{MeV}$, corresponding to $n_{f}=3$. The value of $\Delta S_{\text {ren }}$ should be considered as an estimate of the intrinsic ambiguity in the result for the resummed series $S_{\text {res }}$.

We observe that for the ratios $m_{b} / \bar{m}_{b}\left(m_{b}\right)$ and $f_{B}^{\text {stat }} / f_{B^{*}}^{\text {stat }}$, where the BLM prescription gives very low scales and the corrections to the BLM scheme are large, the resummation leads to results similar to the two-loop approximation. Thus, higher-order corrections are smaller than indicated by the BLM prescription. Nevertheless, in these cases the ambiguity due to the presence of the nearby infrared renormalon at $u=1 / 2$ is quite significant. For the matching constants $\eta_{V}$ and $\eta_{A}$ the resummation follows the tendency indicated by the BLM scheme, and the results are close to what one obtains taking into account the leading correction to that scheme. The renormalon ambiguities are smaller in this case, since the nearest infrared renormalon pole is located at $u=1$.

Another way of comparing our resummation to the BLM scheme is to define, for each series, a scale $\mu_{*}$ such that the one-loop correction evaluated using that scale reproduces the resummed series. Hence, we write

$$
\begin{aligned}
\frac{m_{b}}{m_{b}^{\mathrm{R}}} & =1+\left(\frac{1}{2}+r\right) \frac{\alpha_{s}\left(\mu_{*}\right)}{\pi}, \\
\frac{f_{B}^{\text {stat }}}{f_{B^{*}}^{\text {stat }}} & =1+\frac{2}{3} \frac{\alpha_{s}\left(\mu_{*}\right)}{\pi},
\end{aligned}
$$


Table 5: Comparison of the BLM scale with the scale $\mu_{*}$ corresponding to the partial resummation of the series. All values refer to the $\overline{\mathrm{MS}}$ scheme. In the $\mathrm{V}$ scheme, the scales are larger by a factor $e^{5 / 6} \simeq 2.3$

\begin{tabular}{l|cccc}
\hline \hline & $M$ & $\mu_{\mathrm{BLM}} / M$ & $\mu_{*} / M$ & $\mu_{*} / \mu_{\mathrm{BLM}}$ \\
\hline$m_{b} / m_{b}^{\mathrm{R} 1}$ & $m_{b}$ & 0.131 & 0.195 & 1.49 \\
$m_{b} / m_{b}^{\mathrm{R} 2}$ & $m_{b}$ & 0.435 & 0.378 & 0.87 \\
$m_{b} / \bar{m}_{b}\left(m_{b}\right)$ & $m_{b}$ & 0.096 & 0.179 & 1.86 \\
$f_{B}^{\text {stat }} / f_{B^{*}}^{\text {stat }}$ & $m_{b}$ & 0.205 & 0.259 & 1.26 \\
$\eta_{V}$ & $\sqrt{m_{b} m_{c}}$ & 0.920 & 0.690 & 0.75 \\
$\eta_{V}-\eta_{A}$ & $\sqrt{m_{b} m_{c}}$ & 0.628 & 0.418 & 0.67 \\
\hline \hline & \\
$\eta_{V}-$ & $\eta_{A}=\frac{2}{3} \frac{\alpha_{s}\left(\mu_{*}\right)}{\pi}$.
\end{tabular}

In Table 5 these scales are compared to the BLM scales. Note that in the cases with the lowest BLM scale, namely for the ratios of masses and decay constants, the resummation leads to a larger scale $\mu_{*}>\mu_{\mathrm{BLM}}$.

Our next goal is to obtain an estimate for the relative and absolute size of the short- and long-distance contributions to the various quantities considered above. To this end we introduce a factorization scale $\lambda=1 \mathrm{GeV}$ and evaluate separately the two integrals $S_{\text {sd }}$ and $S_{\text {ld }}$ defined in (30). The factorization scale is chosen such that the value of the coupling constant $\alpha_{s}\left(e^{C} \lambda^{2}\right) / \pi \simeq 0.25$ is still in the perturbative regime (note that $e^{C / 2} \lambda \simeq 0.43 \mathrm{GeV}$ in the $\overline{\mathrm{MS}}$ scheme). In order to model the long-distance contribution, we guess a "realistic" behaviour of the coupling constant in the infrared region. We use a modified version of the running coupling constant, which exhibits freezing for $\mu \rightarrow 0$ :

$$
\alpha_{s}\left(e^{C} \mu^{2}\right)=\frac{4 \pi}{\beta_{0} \ln \left(c+\mu^{2} / \Lambda_{V}^{2}\right)} .
$$

We shall investigate the cases where $c$ is adjusted so that $\alpha_{s}(0)=1,2$ and 4 (in the $\overline{\mathrm{MS}}$ scheme), and interpret the dependence of the results on $c$ as a measure of the model dependence. In Table 6 we compare the sum $S_{\text {tot }}=S_{\text {sd }}+S_{\text {ld }}$ to the "perturbative" resummation obtained using the one-loop running coupling constant regulated with a principle value prescription. We also give the results for the short- and long-distance contributions separately. For each case, we write the long-distance contribution as a power correction, $\left|S_{\mathrm{ld}}\right|=(\Lambda / M)^{2 k}$, and quote the 
Table 6: Comparison of resummed "perturbative" series $S_{\text {res }}$ with a model calculation of the full series including long-distance effects, $S_{\text {tot }}=S_{\text {sd }}(\lambda)+S_{\mathrm{ld}}(\lambda)$. The notation is such that the main values given for $S_{\text {tot }}, S_{\text {ld }}$ and $\Lambda$ correspond to $\alpha_{s}(0)=2$, whereas the corrections indicated as super- and subscripts refer to $\alpha_{s}(0)=1$ and 4 , respectively. We use $\lambda=1 \mathrm{GeV}$ for the factorization scale.

\begin{tabular}{l|cc|ccc}
\hline \hline & $S_{\text {res }}$ & $S_{\text {tot }}$ & $S_{\text {sd }}(\lambda)$ & $S_{\text {ld }}(\lambda)$ & $\Lambda(\lambda)[\mathrm{MeV}]$ \\
\hline$m_{b} / m_{b}^{\mathrm{R} 1}$ & 0.178 & $0.243_{+0.046}^{-0.039}$ & 0.138 & $0.105_{+0.046}^{-0.039}$ & $501_{+221}^{-186}$ \\
$m_{b} / m_{b}^{\mathrm{R} 2}$ & 0.304 & $0.369_{+0.046}^{-0.039}$ & 0.264 & $0.105_{+0.046}^{-0.039}$ & $501_{+221}^{-186}$ \\
$m_{b} / \bar{m}_{b}\left(m_{b}\right)$ & 0.188 & $0.252_{+0.046}^{-0.039}$ & 0.147 & $0.105_{+0.046}^{-0.039}$ & $501_{+221}^{-186}$ \\
$f_{B}^{\text {stat }} / f_{B^{*}}^{\text {stat }}$ & 0.076 & $0.119_{+0.029}^{-0.023}$ & 0.057 & $0.062_{+0.029}^{-0.023}$ & $300_{+138}^{-111}$ \\
$\eta_{V}$ & 0.023 & $0.020_{-0.001}^{+0.001}$ & 0.024 & $-0.004_{-0.001}^{+0.001}$ & $154_{+25}^{-21}$ \\
$\eta_{V}-\eta_{A}$ & 0.081 & $0.083_{+0.005}^{-0.006}$ & 0.064 & $0.019_{+0.005}^{-0.006}$ & $363_{+47}^{-67}$ \\
\hline \hline
\end{tabular}

value of the low-energy scale $\Lambda$. We observe that the long-distance contribution is large, as big as the short-distance one, for the ratios of the heavy quark masses (in the schemes $\mathrm{R} 1$ and $\overline{\mathrm{MS}}$ ) and for the ratio of the decay constants. The reason is that the nearest infrared renormalon is located at $u=1 / 2$, leading to nonperturbative corrections suppressed by only one power of the large mass scale $m_{b}$. In general, the scales $\Lambda$ associated with the long-distance contributions are typical low-energy scales of QCD.

In Figs. 5 and 6 we show our predictions for the short-distance contributions to the quantities $m_{b} / \bar{m}_{b}\left(m_{b}\right), f_{B} / f_{B^{*}}, \eta_{V}$ and $\eta_{A}$ as a function of the factorization scale in the range $\lambda_{0}<\lambda<M$, where $\lambda_{0} \simeq 0.823 \mathrm{GeV}$ is the point where $\alpha_{s}\left(e^{C} \lambda_{0}^{2}\right)=1$. These results will become useful once non-perturbative calculations of matrix elements performed with a hard ultraviolet cutoff become available. The idea to introduce a hard factorization scale to organize the heavy quark expansion has been put forward recently by Bigi et al. [29]. Our approach provides a consistent framework to implement this proposal. Let us illustrate this with two examples of phenomenological importance. The first is the ratio of the physical meson decay constants $f_{B}$ and $f_{B^{*}}$, which is related to the ratio defined in the static limit by

$$
\frac{f_{B}}{f_{B^{*}}}=\frac{f_{B}^{\text {stat }}}{f_{B^{*}}^{\text {stat }}}+\frac{A}{m_{b}}+O\left(1 / m_{b}^{2}\right)
$$

The non-perturbative parameter $A$ can be defined in terms of hadronic matrix elements of dimension-four operators in the heavy quark effective theory [34], which have to be estimated using non-perturbative techniques such as lattice 


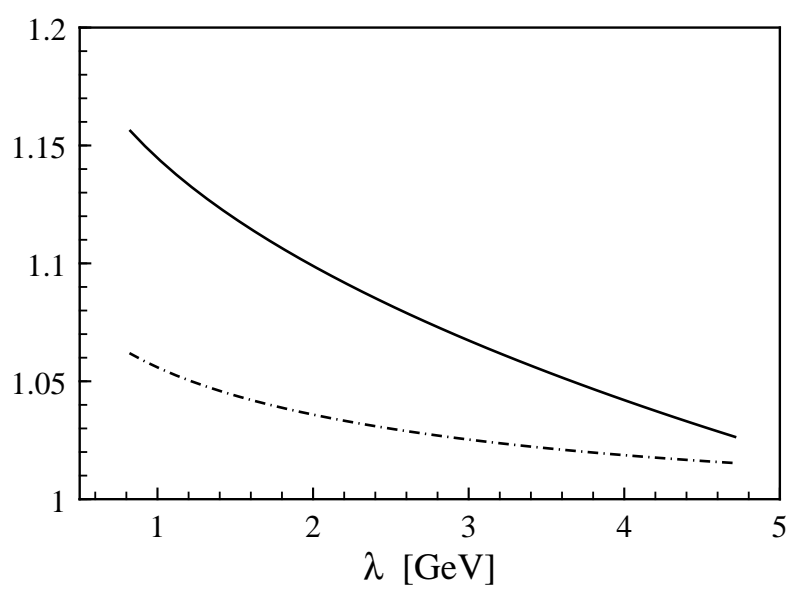

Figure 5: Short-distance contributions to $m_{b}(\lambda) / \bar{m}_{b}\left(m_{b}\right)$ (solid line) and $\left(f_{B} / f_{B^{*}}\right)(\lambda)$ (dashed-dotted line) as a function of the factorization scale.

gauge theory or QCD sum rules. These matrix elements are linearly ultraviolet divergent. If in the calculation of $A$ one introduces a hard ultraviolet cutoff $\lambda$ in the same way as it was done for the perturbative calculation in the static limit, these matrix elements contain those long-distance contributions excluded in the short-distance calculation. Hence, one obtains

$$
\frac{f_{B}}{f_{B^{*}}}=1+S_{\mathrm{sd}}\left(m_{b}^{2}, \lambda\right)+\frac{A(\lambda)}{m_{b}}+O\left(1 / m_{b}^{2}\right) \equiv \frac{f_{B}}{f_{B^{*}}}(\lambda)+\frac{A(\lambda)}{m_{b}}+O\left(1 / m_{b}^{2}\right) .
$$

Our resummed expression for the short-distance contribution is

$$
S_{\mathrm{sd}}\left(m_{b}^{2}, \lambda\right)=\int_{\lambda^{2} / m_{b}^{2}}^{\infty} \mathrm{d} \tau \widehat{w}_{2}(\tau) \frac{\alpha_{s}\left(\tau e^{C} m_{b}^{2}\right)}{4 \pi},
$$

where the distribution function $\widehat{w}_{2}(\tau)$ has been given in (55). The $\lambda$-dependence in (73) cancels between the short- and long-distance pieces. Let us note that often heavy quark expansions such as (72) are written down using dimensional regularization, in which case there is no clear separation between short- and longdistance contributions. Then the perturbative series contains an infrared renormalon at $u=1 / 2$, which is exactly compensated by an ultraviolet renormalon in the parameter $A$ [30]. Again, the sum of all perturbative and non-perturbative terms is unambiguous.

Our second example involves the matching factor $\eta_{A}$ for a flavour-changing axial vector current containing two heavy quarks. From the measurement of the recoil spectrum in the semi-leptonic decay $B \rightarrow D^{*} \ell \bar{\nu}_{\ell}$ one can extract the product $\left|V_{\mathrm{cb}}\right| \mathcal{F}(1)$, where $\mathcal{F}(1)$ denotes the value of the hadronic form factor of the decay at the kinematic point of zero recoil [36]. This form factor is usually 


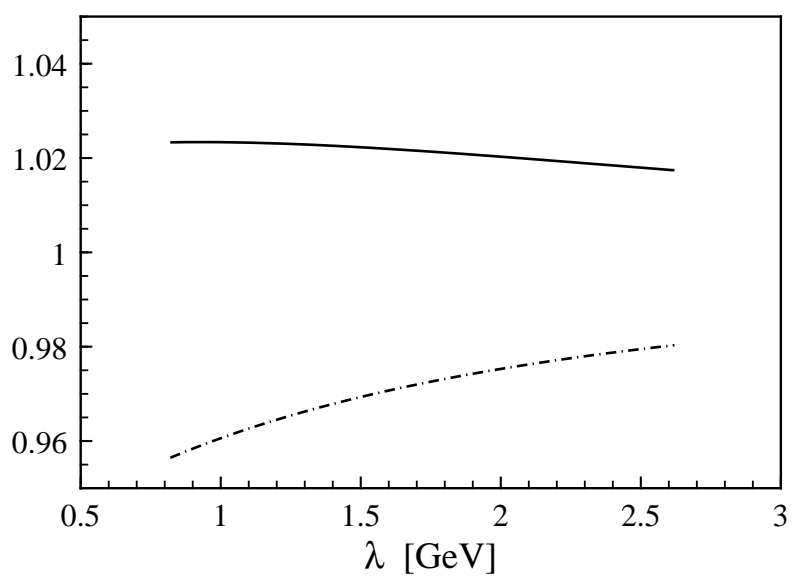

Figure 6: Short-distance contributions to $\eta_{V}(\lambda)$ (solid line) and $\eta_{A}(\lambda)$ (dashed-dotted line) as a function of the factorization scale.

factorized in the form $\mathcal{F}(1)=\eta_{A}\left(1+\delta_{1 / m^{2}}\right)$, where the quantity $\delta_{1 / m^{2}}$ represents non-perturbative power corrections, which from a conceptual point of view cannot be distinguished from the long-distance contributions to $\eta_{A}$. Hence, to separate short- and long-distance effects properly one should again introduce a factorization scale, so that $\eta_{A}(\lambda)$ contains all short-distance contributions, while $\delta_{1 / m^{2}}(\lambda)$ accounts for long-distance effects. The problem is that so far all calculations of $\delta_{1 / m^{2}}$ were based on phenomenological approaches that do not account for the $\lambda$-dependence [40]- [42]. Therefore, we have to rely on a reasonable guess for the factorization scale when we combine the most recent estimate $\delta_{1 / m^{2}}=-(5.5 \pm 2.5) \%$ [36] with our short-distance calculation. From Fig. 6 we find that $0.955<\eta_{A}(\lambda)<0.975$ for $0.8 \mathrm{GeV}<\lambda<2 \mathrm{GeV}$, which we consider a conservative range of values for the factorization scale. This yields

$$
\mathcal{F}(1)=\eta_{A}(\lambda)\left[1+\delta_{1 / m^{2}}(\lambda)\right]=0.91 \pm 0.03
$$

which is $2 \%$ larger than the result obtained in Ref. [42], and $2 \%$ smaller than the value quoted in Ref. [36]. The corresponding shift in the value of $\left|V_{\mathrm{cb}}\right|$ is at the level of $10^{-3}$.

\section{Correlator of light vector currents}

As an important example not related to heavy quarks, we investigate the perturbative expansion of the correlator of two vector currents in the euclidean region $\left(Q^{2}=-q^{2}>0\right)$

$$
i \int \mathrm{d}^{4} x e^{i q \cdot x}\left\langle 0\left|T\left\{j^{\mu}(x), j^{\nu}(0)\right\}\right| 0\right\rangle=\left(q^{\mu} q^{\nu}-q^{2} g^{\mu \nu}\right) \Pi\left(Q^{2}\right),
$$


where $j^{\mu}=\bar{q} \gamma^{\mu} q$. For simplicity we shall consider massless quarks. The momentum transfer $Q^{2}$ provides the large mass scale. The derivative of $\Pi\left(Q^{2}\right)$ with respect to $Q^{2}$ is ultraviolet convergent. As usual, we define the $D$-function

$$
D\left(Q^{2}\right)=4 \pi^{2} Q^{2} \frac{\mathrm{d} \Pi\left(Q^{2}\right)}{\mathrm{d} Q^{2}}=1+S_{D}\left(Q^{2}\right) .
$$

In the large- $\beta_{0}$ limit, the Borel transform of the perturbative series for $S_{D}\left(Q^{2}\right)$ is well known [27, 43]:

$$
\begin{aligned}
\widehat{S}_{D}(u) & =\frac{32 C_{F}}{2-u} \sum_{k=2}^{\infty} \frac{(-1)^{k} k}{\left[k^{2}-(1-u)^{2}\right]^{2}} \\
& =3 C_{F}\left\{1+\left(\frac{23}{6}-4 \zeta(3)\right) u+(9-6 \zeta(3)) u^{2}+O\left(u^{3}\right)\right\}
\end{aligned}
$$

To obtain the corresponding distribution function we start from the first relation in (20) and set $u_{0}=1$. This gives

$$
\widehat{w}_{D}(\tau)=\frac{8 C_{F}}{\pi} \sum_{k=2}^{\infty}(-1)^{k} \frac{\mathrm{d}}{\mathrm{d} k} \frac{1}{k^{2}-1} \int_{-\infty}^{\infty} \mathrm{d} r e^{i r \ln \tau}\left(\frac{1+i r}{r^{2}+k^{2}}-\frac{1}{1-i r}\right) .
$$

The integral can be performed closing the integration contour at infinity; however, it is necessary to distinguish the cases $\ln \tau>0$ and $\ln \tau<0$. We find

$$
\begin{aligned}
& \widehat{w}_{D}(\tau)=8 C_{F}\left\{\left(\frac{7}{4}-\ln \tau\right) \tau+(1+\tau)\left[L_{2}(-\tau)+\ln \tau \ln (1+\tau)\right]\right\} ; \tau<1, \\
& \widehat{w}_{D}(\tau)=8 C_{F}\left\{1+\ln \tau+\left(\frac{3}{4}+\frac{1}{2} \ln \tau\right) \frac{1}{\tau}\right. \\
&\left.+(1+\tau)\left[L_{2}\left(-\tau^{-1}\right)-\ln \tau \ln \left(1+\tau^{-1}\right)\right]\right\} ; \quad \tau>1,
\end{aligned}
$$

where $L_{2}(x)=-\int_{0}^{x} \frac{\mathrm{d} y}{y} \ln (1-y)$ is the dilogarithm function. The distribution function and its first three derivatives are continuous at $\tau=1$, but higher derivatives are not. The asymptotic behaviour for $\tau \rightarrow 0$ is given by

$$
\widehat{w}_{D}(\tau)=6 C_{F} \tau+O\left(\tau^{2}\right),
$$

corresponding to the infrared renormalon pole at $u=2$ in the Borel transform in (78). The location of this renormalon is consistent with the structure of the OPE for $\Pi\left(Q^{2}\right)$, in which non-perturbative corrections appear first t at order $1 / Q^{4}$. This will be discussed in more detail below. For large values of $\tau$ the distribution function behaves like $\ln \tau / \tau^{2}$, so that the integral over the distribution function is ultraviolet convergent.

\footnotetext{
${ }^{1}$ The question whether there is an infrared renormalon at $u=1$ in real QCD (beyond the large- $\beta_{0}$ approximation) is, however, not completely settled [26, 44].
} 
Table 7: Parameters obtained from the distribution function corresponding to the $D$-function. We use $Q_{1}^{2}=2 \mathrm{GeV}^{2}$ and $Q_{2}^{2}=(20 \mathrm{GeV})^{2}$.

\begin{tabular}{c|cccccc}
\hline \hline & $\mu_{\mathrm{BLM}}^{V}$ & $\mu_{\mathrm{BLM}}^{\overline{\mathrm{MS}}}$ & $\alpha_{s}\left(\mu_{\mathrm{BLM}}^{2}\right)$ & $\sigma_{\tau}$ & $\Delta$ & $\delta_{\mathrm{BLM}}$ \\
\hline$D\left(Q_{1}^{2}\right)$ & $1.628 \sqrt{Q_{1}^{2}}$ & $0.708 \sqrt{Q_{1}^{2}}$ & 0.40 & 1.620 & 2.625 & 0.22 \\
$D\left(Q_{2}^{2}\right)$ & $1.628 \sqrt{Q_{2}^{2}}$ & $0.708 \sqrt{Q_{2}^{2}}$ & 0.17 & 1.620 & 2.625 & 0.03 \\
\hline \hline
\end{tabular}

Using the above result, one can compute

$$
\begin{aligned}
N & =\frac{3}{4} C_{F}=1, \\
\langle\ln \tau\rangle & =4 \zeta(3)-\frac{23}{6} \simeq 0.975, \\
\left\langle\ln ^{2} \tau\right\rangle & =18-12 \zeta(3) \simeq 3.575 .
\end{aligned}
$$

The resulting values for the BLM scale and for the parameters $\sigma_{\tau}, \Delta$ and $\delta_{\mathrm{BLM}}$ are shown in Table 7 for both a small and a large value of $Q^{2}$. A graphical representation of the distribution function is given in Fig. 7 .

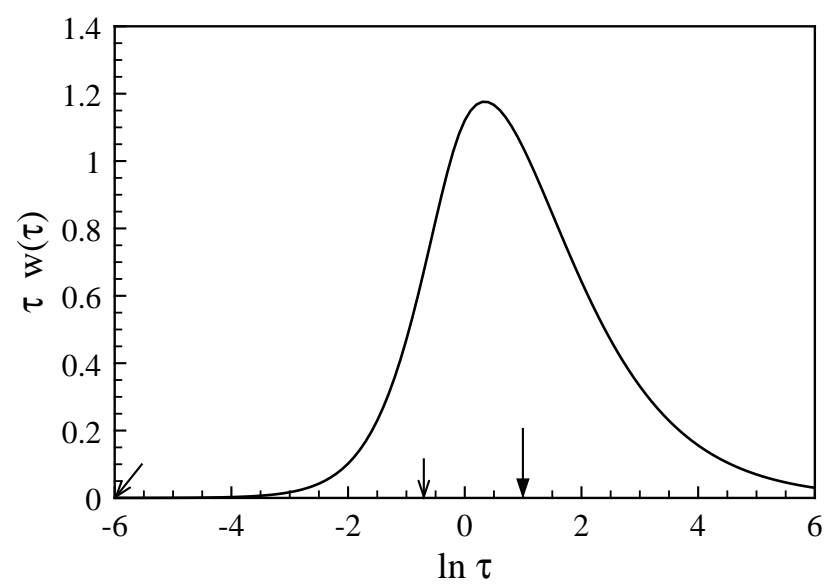

Figure 7: Distribution function for the $D$-function. The long arrow shows the BLM scale. The short arrows indicate the factorization point $\tau=\lambda^{2} / Q^{2}$ for $Q_{1}^{2}=2 \mathrm{GeV}^{2}$ (right) and $Q_{2}^{2}=(20 \mathrm{GeV})^{2}$ (left).

For completeness we also discuss the resummation for the correlator $\Pi\left(Q^{2}\right)$ itself. To obtain it, we integrate (77) and use the fact that $S_{D}\left(Q^{2}\right)$ depends on 
$Q^{2}$ only through the running coupling constant to find

$$
4 \pi^{2}\left[\Pi\left(Q^{2}\right)-\Pi\left(Q_{0}^{2}\right)\right]_{\mathrm{res}}=\ln \frac{Q^{2}}{Q_{0}^{2}}+\frac{1}{2 \pi} \int_{0}^{\infty} \mathrm{d} \tau \widehat{w}_{D}(\tau) \int_{\alpha_{s}\left(\tau e^{C} Q_{0}^{2}\right)}^{\alpha_{s}\left(\tau e^{C} Q^{2}\right)} \mathrm{d} \alpha_{s} \frac{\alpha_{s}}{\beta\left(\alpha_{s}\right)},
$$

where $\beta\left(\alpha_{s}\right)=\mathrm{d} \alpha_{s}\left(\mu^{2}\right) / \mathrm{d} \ln \mu$ is the $\beta$-function, and $Q_{0}^{2}$ is some arbitrary reference scale, which serves to subtract the ultraviolet divergence of $\Pi\left(Q^{2}\right)$. To obtain the distribution function for $\Pi\left(Q^{2}\right)$ it is sufficient to use the one-loop $\beta$-function,

$$
\beta\left(\alpha_{s}\right)=-\frac{\beta_{0}}{2 \pi} \alpha_{s}^{2}
$$

which leads to

$$
4 \pi^{2}\left[\Pi\left(Q^{2}\right)-\Pi\left(Q_{0}^{2}\right)\right]_{\mathrm{res}}=\ln \frac{Q^{2}}{Q_{0}^{2}}-\frac{1}{\beta_{0}} \int_{0}^{\infty} \mathrm{d} \tau \widehat{w}_{D}(\tau) \ln \frac{\alpha_{s}\left(\tau e^{C} Q^{2}\right)}{\alpha_{s}\left(\tau e^{C} Q_{0}^{2}\right)} .
$$

Using an integration by parts we can bring this into the standard form of the distribution function representation:

$$
4 \pi^{2}\left[\Pi\left(Q^{2}\right)-\Pi\left(Q_{0}^{2}\right)\right]_{\mathrm{res}}=\ln \frac{Q^{2}}{Q_{0}^{2}}+\int_{0}^{\infty} \mathrm{d} \tau \widehat{w}_{\Pi}(\tau)\left\{\frac{\alpha_{s}\left(\tau e^{C} Q^{2}\right)}{4 \pi}-\frac{\alpha_{s}\left(\tau e^{C} Q_{0}^{2}\right)}{4 \pi}\right\},
$$

with the distribution function

$$
\widehat{w}_{\Pi}(\tau)=-\frac{1}{\tau} \int_{0}^{\tau} \mathrm{d} \tau^{\prime} \widehat{w}_{D}\left(\tau^{\prime}\right)
$$

Performing the integral gives

$$
\begin{aligned}
\widehat{w}_{\Pi}(\tau)=-4 C_{F}\left\{1-\ln \tau+\left(\frac{5}{2}-\frac{3}{2} \ln \tau\right) \tau\right. \\
\left.+\frac{(1+\tau)^{2}}{\tau}\left[L_{2}(-\tau)+\ln \tau \ln (1+\tau)\right]\right\} ; \quad \tau<1, \\
\widehat{w}_{\Pi}(\tau)=-4 C_{F}\left\{1+\ln \tau+\left(\frac{5}{2}+\frac{3}{2} \ln \tau\right) \frac{1}{\tau}\right. \\
\left.+\frac{(1+\tau)^{2}}{\tau}\left[L_{2}\left(-\tau^{-1}\right)-\ln \tau \ln \left(1+\tau^{-1}\right)\right]\right\} ; \quad \tau>1 .
\end{aligned}
$$

The same result can also be obtained directly by starting from the Borel transform of the correlator $\Pi\left(Q^{2}\right)$. The asymptotic behaviour for small values of $\tau$ is

$$
\widehat{w}_{\Pi}(\tau)=-3 C_{F} \tau+O\left(\tau^{2}\right)
$$


Table 8: Various approximations for the perturbative contribution to the $D$ function. As previously $Q_{1}^{2}=2 \mathrm{GeV}^{2}$ and $Q_{2}^{2}=(20 \mathrm{GeV})^{2}$.

\begin{tabular}{c|cccccc}
\hline \hline & $S_{1-\text { loop }}$ & $S_{2-\text { loop }}$ & $S_{\mathrm{BLM}}$ & $S_{\mathrm{BLM}^{*}}$ & $S_{\text {res }}$ & $\Delta S_{\text {ren }}$ \\
\hline$D\left(Q_{1}^{2}\right)$ & 0.107 & 0.125 & 0.128 & 0.156 & 0.164 & 0.006 \\
$D\left(Q_{2}^{2}\right)$ & 0.050 & 0.054 & 0.054 & 0.055 & 0.055 & $1.5 \times 10^{-7}$ \\
\hline \hline
\end{tabular}

Table 9: Comparison of the BLM scale with the scale $\mu_{*}$ corresponding to the full resummation of the series. All values refer to the $\overline{\mathrm{MS}}$ scheme.

\begin{tabular}{c|ccc}
\hline \hline & $\mu_{\mathrm{BLM}} / \sqrt{Q^{2}}$ & $\mu_{*} / \sqrt{Q^{2}}$ & $\mu_{*} / \mu_{\mathrm{BLM}}$ \\
\hline$D\left(Q_{1}^{2}\right)$ & 0.708 & 0.485 & 0.685 \\
$D\left(Q_{2}^{2}\right)$ & 0.708 & 0.621 & 0.878 \\
\hline \hline
\end{tabular}

corresponding again to an infrared renormalon at $u=2$. For large values of $\tau$ the distribution function falls off like $1 / \tau$, in accordance with the logarithmic ultraviolet divergence of the unsubtracted correlator.

Let us now turn to the numerical analysis of our results. In Table 8 we show the various approximations to the series $S_{D}\left(Q^{2}\right)$, as defined in (69). We also quote values for the renormalon ambiguity

$$
\Delta D_{\text {ren }}=\left(\Delta S_{D}\right)_{\text {ren }}=\frac{8}{\beta_{0}}\left(\frac{\Lambda_{V}^{2}}{Q^{2}}\right)^{2} .
$$

It is apparent that the effect of the resummation is more pronounced in the case where $Q^{2}$ is low. In this case there are significant corrections to the BLM scheme. For the resummed series $S_{\text {res }}\left(Q^{2}\right)$ we define a scale $\mu_{*}$ so that

$$
D\left(Q^{2}\right)=1+\frac{\alpha_{s}\left(\mu_{*}^{2}\right)}{\pi} .
$$

This scale is compared to the BLM scale in Table 9. Finally, in Table 10 we evaluate the short- and long-distance contributions to the $D$-function introducing a factorization scale $\lambda=1 \mathrm{GeV}$.

From the asymptotic behaviour of the distribution function in (81) we conclude that the long-distance contribution to the perturbative series scales like

$$
S_{\mathrm{ld}}\left(Q^{2}, \lambda\right)=\frac{\Lambda^{4}(\lambda)}{\left(Q^{2}\right)^{2}}
$$


Table 10: Comparison of resummed "perturbative" series $S_{\text {res }}$ with a model calculation of the full series including long-distance effects, $S_{\text {tot }}=S_{\mathrm{sd}}(\lambda)+S_{\mathrm{ld}}(\lambda)$. We use $\lambda=1 \mathrm{GeV}$ for the factorization scale.

\begin{tabular}{c|cc|ccc}
\hline \hline & $S_{\text {res }}$ & $S_{\text {tot }}$ & $S_{\text {sd }}(\lambda)$ & $S_{\text {ld }}(\lambda)$ & $\Lambda(\lambda)[\mathrm{MeV}]$ \\
\hline$D\left(Q_{1}^{2}\right)$ & 0.164 & $0.146_{+0.005}^{-0.009}$ & 0.118 & $0.028_{+0.005}^{-0.009}$ & $581_{+23}^{-56}$ \\
$D\left(Q_{2}^{2}\right)$ & 0.005 & 0.005 & 0.005 & $(1.6 \pm 0.2) \times 10^{-6}$ & $713_{+16}^{-31}$ \\
\hline \hline
\end{tabular}

It should be combined with non-perturbative contributions of the same magnitude. For the euclidean correlator the OPE of the current product $j^{\mu}(x) j^{\nu}(0)$ provides the framework for a systematic incorporation of non-perturbative effects. At order $1 /\left(Q^{2}\right)^{2}$ these effects are parametrized by the gluon condensate [45]. Hence, to this order we may write

$$
\begin{aligned}
D\left(Q^{2}\right) & =1+S_{\mathrm{sd}}\left(Q^{2}, \lambda\right)+\frac{\Lambda^{4}(\lambda)}{\left(Q^{2}\right)^{2}}+\frac{2 \pi}{3\left(Q^{2}\right)^{2}}\left\langle\alpha_{s} G^{2}\right\rangle+\ldots \\
& \equiv 1+S_{\mathrm{sd}}\left(Q^{2}, \lambda\right)+\frac{2 \pi}{3\left(Q^{2}\right)^{2}}\left\langle\alpha_{s} G^{2}\right\rangle(\lambda)+\ldots
\end{aligned}
$$

where the last equation defines the scale-dependent condensate

$$
\left\langle\alpha_{s} G^{2}\right\rangle(\lambda)=\left\langle\alpha_{s} G^{2}\right\rangle+\frac{3}{2 \pi} \Lambda^{4}(\lambda)
$$

For $\lambda=1 \mathrm{GeV}$, we find from Table 10 that the "perturbative" contribution to the gluon condensate is about $0.1 \mathrm{GeV}^{4}$, which is of the same order of magnitude as the "genuine" gluon condensate $\left\langle\alpha_{s} G^{2}\right\rangle$ 45. In many practical applications of the OPE, and in particular in the phenomenology of the QCD sum rules, it is assumed that the "perturbative" contributions to the vacuum condensates are much smaller than the "genuine" values of the condensates and can be neglected [48]. Our result (94) provides a counter-example to this assertion.

\section{Conclusions}

We have proposed an extension of the BLM scale-setting prescription, which resums certain vacuum polarization insertions to all orders in perturbation theory. Our approach is equivalent to performing one-loop calculations with a running coupling constant, thereby including much of the non-trivial asymptotic behaviour of a perturbative series. The representation of the resummed series as an integral over the running coupling constant with a distribution function, as shown in (22), provides an intuitive picture of the distribution of virtualities in 
a one-loop calculation. Much insight can be gained from the knowledge of the distribution function. Its behaviour for large and small values of the scale parameter $\tau$ is related in a direct way to the ultraviolet and infrared properties of the series. Moments of the distribution function determine the size of higher-order coefficients.

By summing an infinite set of diagrams our scheme reaches beyond perturbation theory. In particular, it provides a clear separation of short- and longdistance effects. In any finite-order perturbative calculation non-perturbative effects are implicitly present due to low-momentum contributions in Feynman diagrams, but are not visible as they are exponentially small in the coupling constant. Yet perturbation theory "knows" about these contributions in the form of infrared renormalon singularities, which make a perturbative series non Borel summable. This means that attempts to resum the series will lead to unavoidable ambiguities. In our scheme the long-distance contributions can be explicitly separated, since it is possible to introduce a hard momentum cutoff in a natural way. The size of the long-distance contributions and their dependence on the large mass scale of the problem is determined by the asymptotic behaviour of the distribution function in the infrared region.

Our approach offers several conceptual advantages over the BLM scheme. In particular, it is additive and works in cases where the BLM scale is low. We have emphasized that the value of the BLM scale alone cannot always be taken as an indicator of the size of higher-order corrections or the rate of convergence of a perturbative series. Except in cases where the distribution function is very narrow, it is better to deduce this information from the distribution function, which properly takes into account the contribution from all mass scales. For instance, the distribution function corresponding to the perturbative series for a quantity which requires a subtraction of ultraviolet divergences typically gets contributions of opposite sign, in which case cancellations may occur at one-loop order resulting in a low value of the BLM scale.

The implementation of our proposal is based on techniques developed for the analysis of renormalon chains. The distribution function can be obtained from the integral relations in (20) by calculating first the Borel transform of a perturbative series with respect to the coupling constant in the limit of large $\beta_{0}$. We have demonstrated this for several one- and two-scale problems in QCD. We find that in many cases the effect of the resummation is quite significant. As shown in Tables 4 and 8, the difference between the resummed result and the two-loop approximation is comparable in magnitude to the difference between the two-loop and the one-loop results, in accordance with the general behaviour expected for asymptotic series. However, we stress that whereas the size of the one- and two-loop coefficients are renormalization-scheme dependent, the result of the resummation is scale- and scheme-independent. We have associated a scale $\mu_{*}$ with the resummed series (using a principle value prescription to regulate the Landau pole in the running coupling constant) and compared this scale to the 
BLM scale. Typically, the two scales can differ by as much as 50\%, which is significant in cases where the BLM scale is low.

Another aspect in our analysis was to investigate the relative size of shortand long-distance contributions to the quantities of interest. Parametrically, longdistance effects are exponentially small in the coupling constant; they have the form of power corrections. In practice, however, they can still be sizeable in some cases. In heavy quark systems, for instance, non-perturbative effects are often only suppressed by one power of the heavy quark mass. Even for the bottom quark they can easily reach a level of $10 \%$, thus being as large as oneloop perturbative corrections. We have introduced a hard factorization scale $\lambda=1 \mathrm{GeV}$ and compared the short-distance contribution to a model calculation of long-distance effects. We find that in some cases the long-distance effects are as big as the short-distance ones. Then no reliable prediction can be obtained based on perturbation theory alone; it is necessary to include non-perturbative effects.

In Ref. [19], we generalize our resummation procedure to the description of cross sections and inclusive decay rates. In the calculation of radiative corrections both virtual and real gluons will have to be considered, and only the sum of their contributions is infrared finite 46, 47]. Clearly, in such a situation one has to generalize the idea of performing a one-loop calculation with a running coupling constant, which was the motivation for our resummation. As a consequence, the "linear" form of the integral representation given in (22) will be replaced by "non-linear" representations, in which instead of the coupling constant $\alpha_{s}$ there appears a function of the coupling constant.

While this paper was in writing, I became aware of a preprint by Beneke and Braun [49], who propose the same generalization of the BLM scheme. Their approach is similar in spirit to the one presented here, although the formalism

differs in technical details. I am grateful to the authors for making their results available to me prior to publication.

\section{Acknowledgements}

It is a pleasure to thank G. Altarelli, P. Ball, J. Ellis, B. Gavela, M. Jamin, A. Kataev, G. Martinelli, P. Nason, O. Pène and A. Pich for useful discussions. 


\section{References}

[1] P.M. Stevenson, Phys. Lett. B 100, 61 (1981); Phys. Rev. D 23, 2916 (1981); Nucl. Phys. B 203, 472 (1982); ibid 231, 65 (1984).

[2] G. Grunberg, Phys. Lett. B 95, 70 (1980); ibid 110, 501 (1982); Phys. Rev. D 29, 2315 (1984).

[3] J. Kubo and S. Sakakibara, Z. Phys. C 14, 345 (1982).

[4] S.J. Brodsky, G.P. Lepage and P.B. Mackenzie, Phys. Rev. D 28, 228 (1983).

[5] A. Dhar and V. Gupta, Phys. Rev. D 29, 2822 (1984).

[6] V.V. Starshenko and R.N. Faustov, JINR Rapid Com. 7, 39 (1985).

[7] V. Gupta, D.V. Shirkov and O.V. Tarasov, Int. J. Mod. Phys. A 6, 3381 (1991).

[8] A.L. Kataev and V.V. Starshenko, CERN preprint CERN-TH.7198/94 (1994) hep-ph 9405249.

[9] H.J. Lu, Phys. Rev. D 45, 1217 (1992).

[10] S.J. Brodsky and H.J. Lu, Phys. Rev. D 48, 3310 (1993); SLAC preprint SLAC-PUB-6481 (1994) hep-ph 9405218.

[11] G. Grunberg and A.L. Kataev, Phys. Lett. B 279, 352 (1992).

[12] G.P. Lepage and P.B. Mackenzie, Phys. Rev. D 48, 2250 (1993).

[13] N. Gray, D.J. Broadhurst, W. Grafe and K. Schilcher, Z. Phys. C 48, 673 (1990).

[14] D.J. Broadhurst and A.G. Grozin, Open University preprint OUT-4102-52 (1994) hep-ph 9410240.

[15] M. Luke, M.J. Savage and M.B. Wise, University of Toronto preprint UTPT 94-24 (1994) hhep-ph 9409287.

[16] K.G. Chetyrkin, A.L. Kataev and F.V. Tkachov, Phys. Lett. B 85, 277 (1979).

[17] M. Dine and J. Sapirstein, Phys. Rev. Lett. 43, 668 (1979).

[18] W. Celmaster and R. Gonsalves, Phys. Rev. Lett. 44, 560 (1980). 
[19] M. Neubert, Resummation of Renormalon Chains for Cross Sections and Inclusive Decay Rates, CERN preprint CERN-TH.7524/94 (1994).

[20] G. 't Hooft, in: The Whys of Subnuclear Physics, Proceedings of the 15th International School on Subnuclear Physics, Erice, Sicily, 1977, edited by A. Zichichi (Plenum Press, New York, 1979), p. 943.

[21] B. Lautrup, Phys. Lett. B 69, 109 (1977).

[22] G. Parisi, Phys. Lett. B 76, 65 (1978); Nucl. Phys. B 150, 163 (1979).

[23] F. David, Nucl. Phys. B 234, 237 (1984); ibid 263, 637 (1986).

[24] A.H. Mueller, Nucl. Phys. B 250, 327 (1985).

[25] V.I. Zakharov, Nucl. Phys. B 385, 452 (1992); M. Beneke and V.I. Zakharov, Phys. Rev. Lett. 69, 2472 (1992).

[26] A.H. Mueller, Phys. Lett. B 308, 355 (1993).

[27] M. Beneke, Phys. Lett. B 307, 154 (1993); Nucl. Phys. B 405, 424 (1993).

[28] M. Beneke and V.M. Braun, Nucl. Phys. B 426, 301 (1994).

[29] I.I. Bigi, M.A. Shifman, N.G. Uraltsev and A.I. Vainshtein, Phys. Rev. D 50, 2234 (1994).

[30] M. Neubert and C.T. Sachrajda, CERN preprint CERN-TH.7312/94 (1994) hep-ph 9407394.

[31] K. Wilson, Phys. Rev. 179, 1499 (1969); Phys. Rev. D 3, 1818 (1971).

[32] H. Georgi, Phys. Lett. B 240, 447 (1990).

[33] For a review, see: M. Neubert, Phys. Rep. 245, 259 (1994); CERN preprint CERN-TH.7225/94, to appear in the Proceedings of the Theoretical Advanced Study Institute in Elementary Particle Physics (TASI-93), Boulder, Colorado, 1993 (World Scientific, Singapore).

[34] M. Neubert, Phys. Rev. D 46, 1076 (1992).

[35] G. Preparata and W.I. Weisberger, Phys. Rev. 175, 1965 (1968).

[36] M. Neubert, Phys. Lett. B 264, 455 (1991); ibid 338, 84 (1994).

[37] M. Neubert, CERN preprint CERN-TH.7454/94 (1994) hep-ph 9409453, to appear in Phys. Lett. B. 
[38] G.P. Korchemsky and A.V. Radyushkin, Nucl. Phys. B 283, 342 (1987); G.P. Korchemsky, Mod. Phys. Lett. A 4, 1257 (1989); G.P. Korchemsky and A.V. Radyushkin, Phys. Lett. B 279, 359 (1992).

[39] A.F. Falk, H. Georgi, B. Grinstein and M.B. Wise, Nucl. Phys. B 343, 1 (1990).

[40] A.F. Falk and M. Neubert, Phys. Rev. D 47, 2965 (1993).

[41] T. Mannel, Phys. Rev. D 50, 428 (1994).

[42] M. Shifman, N.G. Uraltsev and A. Vainshtein, Minnesota preprint TPIMINN-94/13-T (1994) hep-ph 9405207; I. Bigi, M. Shifman, N.G. Uraltsev and A. Vainshtein, Minnesota preprint TPI-MINN-94/12-T (1994) hep-ph 9405410].

[43] D. Broadhurst, Z. Phys. C 58, 339 (1993).

[44] L.S. Brown and L.G. Yaffe, Phys. Rev. D 45, 398 (1992); L.S. Brown, L.G. Yaffe and C. Zhai, Phys. Rev. D 46, 4712 (1992).

[45] M.A. Shifman, A.I. Vainshtein and V.I. Zakharov, Nucl. Phys. B 147, 385 (1979); ibid 147, 448 (1979).

[46] T. Kinoshita, Prog. Theor. Phys. 5, 1045 (1950).

[47] T.D. Li and M. Nauenberg, Phys. Rev. B 133, 1549 (1964).

[48] V.A. Novikov, M.A. Shifman, A.I. Vainshtein and V.I. Zakharov, Nucl. Phys. B 249, 445 (1985).

[49] M. Beneke and V.M. Braun, University of Michigan preprint UM-TH-94-37 (1994) hep-ph 9411229. 